\title{
RNAi-dependent H3K27 methylation is required for heterochromatin formation and DNA elimination in Tetrahymena
}

\author{
Yifan Liu, ${ }^{1,4}$ Sean D. Taverna,,${ }^{1,4}$ Tara L. Muratore, ${ }^{2}$ Jeffrey Shabanowitz, ${ }^{2}$ Donald F. Hunt, ${ }^{2,3}$ \\ and C. David Allis ${ }^{1,5}$ \\ ${ }^{1}$ Laboratory of Chromatin Biology, The Rockefeller University, New York, New York 10021, USA; ${ }^{2}$ Department of \\ Chemistry, University of Virginia, Charlottesville, Virgina 22904, USA; ${ }^{3}$ Department of Pathology, Health Science Center, \\ University of Virginia, Charlottesville, Virginia 22908, USA
}

\begin{abstract}
Methylated H3K27 is an important mark for Polycomb group (PcG) protein-mediated transcriptional gene silencing (TGS) in multicellular eukaryotes. Here a Drosophila $\mathrm{E}(\mathrm{z})$ homolog, EZL1, is characterized in the ciliated protozoan Tetrahymena thermophila and is shown to be responsible for H3K27 methylation associated with developmentally regulated heterochromatin formation and DNA elimination. Importantly, Ezl1p-catalyzed H3K27 methylation occurs in an RNA interference (RNAi)-dependent manner. H3K27 methylation also regulates H3K9 methylation in these processes. Furthermore, an "effector" of programmed DNA elimination, the chromodomain protein Pdd1p, is shown to bind both K27- and K9-methylated H3. These studies provide a framework for an RNAi-dependent, Polycomb group protein-mediated heterochromatin formation pathway in Tetrahymena and underscore the connection between the two highly conserved machineries in eukaryotes.
\end{abstract}

[Keywords: RNA interference; H3K27 methylation; Polycomb group proteins; heterochromatin; DNA elimination]

Supplemental material is available at http://www.genesdev.org.

Received February 21, 2007; revised version accepted May 1, 2007.

Extensive studies, most notably in the fission yeast Schizosaccharomyces pombe, have established RNA interference (RNAi)-dependent H3K9 methylation as a central process for formation of constitutive heterochromatin, by which genes are silenced in pericentromeric regions and other regions containing repetitious DNA sequences (Grewal and Moazed 2003; Martienssen and Moazed 2006). In this pathway, small interfering RNAs (siRNAs) homologous to the repeated sequences are generated by Dicer and associate with an Argonaute homo$\log$ (Hall et al. 2002; Volpe et al. 2002). Via mechanisms not yet fully defined, these siRNAs target histone-modifying activities, in particular, Clr4, a H3K9-specific histone lysine methyltransferase (HKMT) (Nakayama et al. 2001; Noma et al. 2004), to homologous loci. Methylated H3K9 recruits effectors like Swi6 and Chp1 (Hall et al. 2002; Partridge et al. 2002), through direct interaction with the chromodomains of these HP1-like proteins (Bannister et al. 2001; Lachner et al. 2001), leading eventually to the formation of condensed heterochromatin

\footnotetext{
${ }^{4}$ These authors contributed equally to this work.

${ }^{5}$ Corresponding author.

E-MAIL alliscd@mail.rockefeller.edu; FAX (212) 327-7849.

Article is online at http://www.genesdev.org/cgi/doi/10.1101/gad.1544207.
}

structures. These conserved mechanisms are important for maintaining genome integrity as well as transcriptional gene silencing (TGS) in a wide range of eukaryotes (Bernstein and Allis 2005).

Important insights into RNAi-dependent heterochromatin formation have also been gained from studying developmentally regulated DNA elimination in Tetrahymena thermophila. Like most ciliates, Tetrahymena contains a transcriptionally active somatic macronucleus and a transcriptionally inactive germline micronucleus in the same cytoplasmic compartment (Karrer 2000). Dramatic genome reorganization occurs when macronuclei develop from micronuclei during conjugation, the sexual phase of the Tetrahymena life cycle. During a precisely programmed developmental window, $\sim 15 \%$ of the micronuclear genome, mostly moderately repeated sequences, are compacted into cytologically distinct, heterochromatic structures in developing macronuclei (anlagen) (Madireddi et al. 1994) and eventually eliminated from the mature macronuclei (Jahn and Klobutcher 2002). This genome streamlining process functions arguably as the ultimate form of TGS (Coyne et al. 1996; Mochizuki and Gorovsky 2004b).

In keeping with mechanisms underlying RNAi-mediated heterochromatin formation, an RNAi mechanism is 
also involved in DNA elimination. A special class of siRNAs enriched in micronuclear-limited sequences accumulates during conjugation (Mochizuki et al. 2002; Mochizuki and Gorovsky 2004a; Lee and Collins 2006). These siRNAs are produced from double-stranded transcripts synthesized during early conjugation (Chalker and Yao 2001), by the action of DCL1, a Dicer homolog (Malone et al. 2005; Mochizuki and Gorovsky 2005), and their accumulation depends on TWI1, an Argonaute/ PIWI homolog (Mochizuki et al. 2002). Both DCL1 and TWI1 are required for appropriate deposition of methylated H3K9 (Liu et al. 2004; Malone et al. 2005), which associates specifically with micronuclear-limited sequences (Taverna et al. 2002) and is required for their elimination (Liu et al. 2004). Pdd1p and Pdd3p, both abundant conjugation-specific chromodomain-containing proteins, bind methylated H3K9 (Taverna et al. 2002), associate with micronucleus-limited sequences, and are key components of the heterochromatic structures in which DNA elimination occurs (Madireddi et al. 1996; Smothers et al. 1997; Coyne et al. 1999; Nikiforov et al. 2000; Taverna et al. 2002). These observations point to a pathway in which siRNAs target H3K9 methylation and heterochromatin formation to specific chromatin regions (Meyer and Chalker 2007).

Another type of heterochromatin, referred to as facultative heterochromatin, is associated with developmentally regulated TGS and mediated by Polycomb group (PcG) proteins (Ringrose and Paro 2004). Among the most conserved PcG proteins are SET domain-containing $\mathrm{E}(\mathrm{z})$ and homologous HKMTs, which are responsible for H3K27 methylation in Caenorhabditis elegans (Bender et al. 2004), Drosophila (Czermin et al. 2002; Muller et al. 2002), mammals (Cao et al. 2002; Kuzmichev et al. 2002), and Arabidopsis (Bastow et al. 2004; Sung and Amasino 2004). Methylated H3K27, especially in the trimethylated form (H3K27me3), has since been identified as an important mark for facultative heterochromatin, involved in diverse processes like maintenance of the silent state of Hox genes in Drosophila and mammals (Cao et al. 2002), X-chromosome inactivation in female mammals (Erhardt et al. 2003; Plath et al. 2003; Silva et al. 2003; Okamoto et al. 2004), and vernalization in Arabidopsis (Bastow et al. 2004; Sung and Amasino 2004). Polycomb (Pc) and homologous chromodomain proteins specifically interact with H3K27me3 (Cao et al. 2002; Fischle et al. 2003b; Min et al. 2003). This interaction plays an important role in recruiting and stabilizing PcG proteins at the target loci (Fischle et al. 2003b), leading to formation of facultative heterochromatin crucial for TGS.

Recently, evidence has accumulated that hints at a connection between RNAi and H3K27 methylation. Cosuppression at the transcriptional level in Drosophila is Polycomb-dependent (Pal-Bhadra et al. 1999) and affected RNAi-deficient mutants (Pal-Bhadra et al. 2002). RNAi components are also required for nuclear clustering of Polycomb group response elements (Grimaud et al. 2006). In mammalian cells, Agol has been linked with PcG-regulated silencing and Ezh2 [a mammalian
$\mathrm{E}(z)$ homolog]-catalyzed H3K27me3 (Kim et al. 2006). While these results suggest that TGS and facultative heterochromatin formation mediated by $\mathrm{H} 3 \mathrm{~K} 27 \mathrm{me} 3$ may be RNAi dependent, underlying mechanisms remain poorly understood. Here we report the characterization in Tetrahymena of a conjugation-specific H3K27 HKMTs (EZL1) homologous to Drosophila E(z). We further demonstrate that EZL1-dependent H3K27 methylation is required for developmentally regulated DNA elimination and is connected to the RNAi pathway that leads to H3K9 methylation and DNA elimination. We present evidence that $\mathrm{H} 3 \mathrm{~K} 27$ methylation may regulate $\mathrm{H} 3 \mathrm{~K} 9$ methylation, and that both marks are specifically recognized by Pddlp, a chromodomain protein associated with the DNA elimination heterochromatic structures. Our findings lend support to the general view that a highly conserved mechanism underlies diverse heterochromatin-based epigenetic phenomena ranging from DNA elimination in ciliates to developmentally regulated TGS in higher eukaryotes.

\section{Results}

H3K27me3 is a general mark for heterochromatin in Tetrahymena and is associated with developmentally regulated DNA elimination

Recently, we identified methylated H3K27 in Tetrahymena by mass spectrometry analysis and modificationspecific antibodies (Garcia et al. 2007; Taverna et al. 2007). Here we focus on the function(s) of trimethylated H3K27 (H3K27me3), paying particular attention to the process of DNA elimination during conjugation. In Tetrahymena, repressed chromatin is typically found in micronuclei, chromatin bodies in macronuclei, and DNA elimination structures in developing macronuclei (anlagen), also known as DNA elimination bodies (Fig. 1A, red highlights). Immunofluorescence staining with H3K27me3-specific antibodies showed that this modification was associated with all three heterochromatic structures (Fig. 1B). In vegetatively growing cells, H3K27me3 was enriched in the transcriptionally inactive micronucleus (Fig. 1B, first panel), which is cytologically highly condensed, biochemically hypoacetylated (Vavra et al. 1982), and hypomethylated at H3K4 (Strahl et al. 1999). H3K27me3 was present at much lower abundance in the transcriptionally active macronucleus and mostly decorated chromatin bodies (Fig. 1B, first panel, open arrowhead), which are regions of condensed chromatin marked by Hhplp, a HP1 homolog, and associated with transcriptional silencing (Huang et al. 1998, 1999).

In conjugating cells, the focus of this study, H3K27me3 was found in both parental macronuclei (Fig. $1 \mathrm{~B}$, second panel) and developing macronuclei (anlagen). In anlagen, H3K27me3 was initially evenly distributed (Fig. 1B, third panel), but gradually condensed into a pattern resembling DNA elimination structures that are regularly observed with Pddlp antibodies (Fig. 1B, last panel, solid arrowhead). The distribution pattern of H3K27me3 was also confirmed by Western blot analysis 
Liu et al.

A

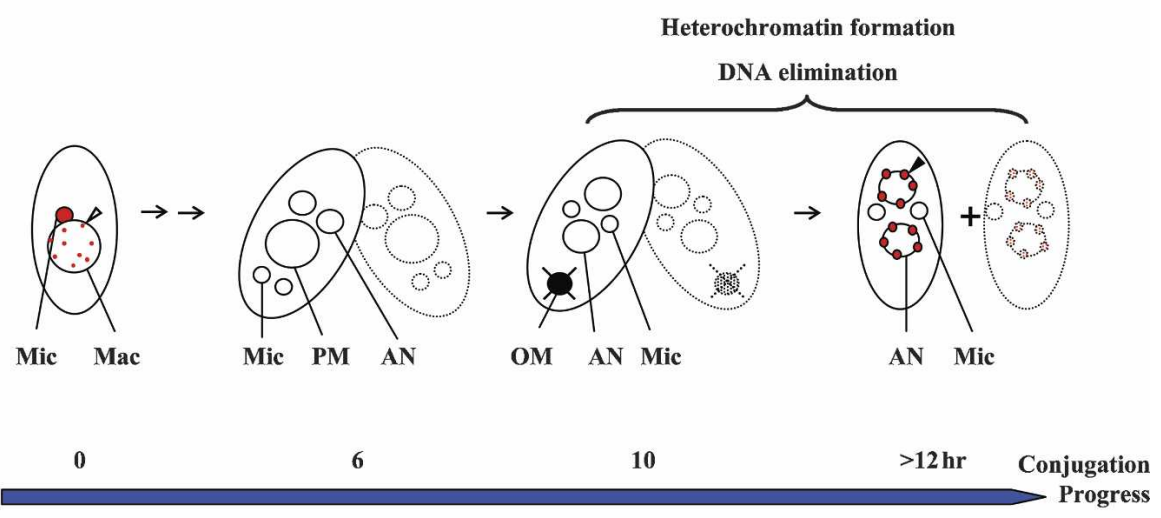

B

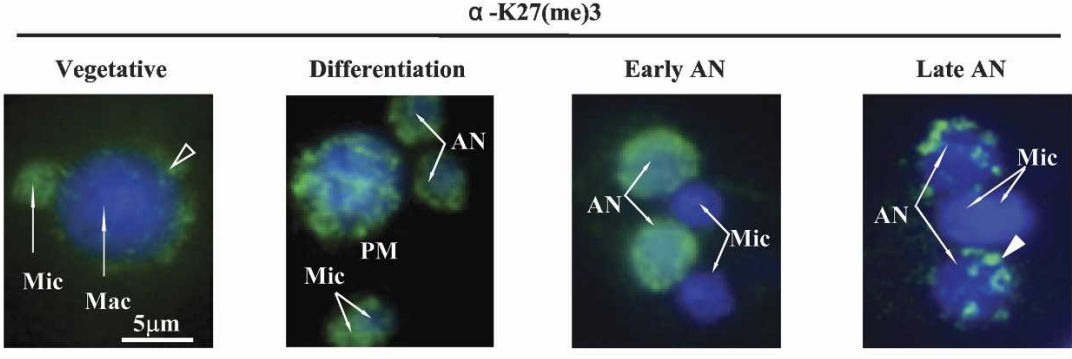

C

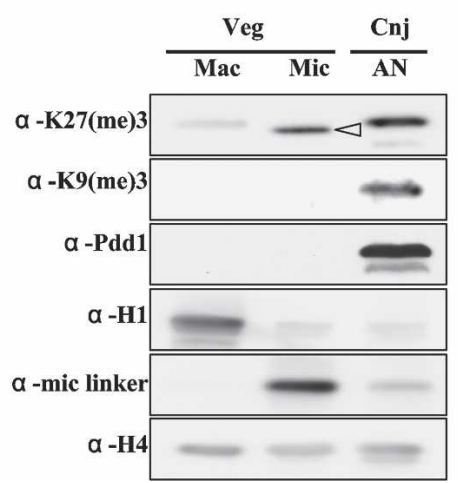

$\mathrm{D}$

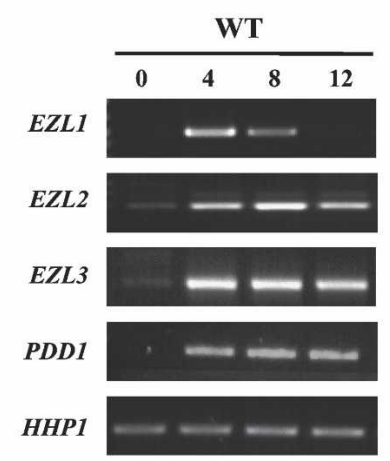

Figure 1. H3K27me3 is a general mark for heterochromatin in Tetrahymena. (A) Schematic representation of key nuclear events in Tetrahymena conjugation. Electron-dense chromatin bodies (open arrowhead) are dispersed in the somatic macronucleus (Mac) of vegetatively growing (nonmating) cells $(0 \mathrm{~h})$. Two Tetrahymena cells of different mating types can pair during conjugation (only one partner is shown in immunofluorescence pictures). During this sexual pathway, the germline micronucleus (Mic) gives rise to two new micronuclei and two developing macronuclei, also referred to as anlagen (AN), supported by transcription from the parental macronuclei (PM) (6 h). As anlagen formation progresses, the old macronucleus (OM) degenerates (10 h). At late conjugation, specialized DNA elimination heterochromatic structures (solid arrowhead) form in anlagen as pairs separate ( $>12 \mathrm{~h}$ ). Heterochromatic structures are highlighted (red). (B) Localization of H3K27me3. Wild-type cells were processed for immunofluorescence staining with H3K27me3specific antibody and counterstained with DAPI. From left to right, typical examples of stained cells are shown as vegetatively growing, micronuclei/anlagen differentiation, and early and late anlagen stages, aligned with their schematic representations in $A$. $(C)$ Differential usage of $\mathrm{H} 3 \mathrm{~K} 27 \mathrm{me} 3$ and H3K9me3 in distinct Tetrahymena nuclei. Acid extracts from purified micronuclei and macronuclei in vegetatively growing cells (Veg) or anlagen isolated from 10-h conjugating cells (Cnj) were resolved on $10 \%$ SDS-PAGE, blotted, and probed with the indicated antibodies. Note that H3K27me3 was only observed in the fast-migrating form of micronuclear H3 (arrowhead), which corresponds to a mature, proteolytically processed H3 (Allis et al. 1980). Another further truncated form was observed in anlagen. $(D)$ Expression of EZLS mRNA during conjugation. Total RNA samples from wild-type and $\triangle E Z L 1$ cells from different conjugation time points $(0,4,8$, and $12 \mathrm{~h}$ post-mixing) were reverse-transcribed and analyzed by PCR with primers specific for EZL1, EZL2, EZL3, PDD1 (a conjugation-specific chromodomain protein), and HHP1 (a gene encoding a chromodomain protein ubiquitously expressed in vegetative and conjugating cells).

of purified nuclei (Fig. 1C). Macronuclei and micronuclei from vegetative cells and anlagen from 10-h conjugating cells were purified (see figure legends for details); Pdd1p,
H1, and micronuclear linker histone served as purity markers for anlagen, macronuclei, and micronuclei, respectively. In comparable amounts of total histones, 
much higher levels of H3K27me3 were detected in micronuclei and anlagen than in macronuclei. In keeping with earlier reports (Taverna et al. 2002), H3K9 methylation was only present in anlagen of conjugating cells. Little or no cross-reaction was observed between H3K27me3 and H3K9me3 antibodies, as confirmed by peptide competition experiments (Supplementary Fig. 1A). These antibody-based results were corroborated by our mass spectrometry analysis, which revealed peptides abundantly decorated with H3K27 methylation in vegetatively growing (data not shown) and conjugating samples (Supplementary Table 1). These data suggest that $\mathrm{H} 3 \mathrm{~K} 27 \mathrm{me} 3$ is a general mark of various heterochromatic structures in Tetrahymena.

\section{$E(z)$ homologs are present in Tetrahymena}

Three putative H3K27-specific HKMTs, EZL1, EZL2, and EZL3, were identified in Tetrahymena genome by their homology with Drosophila E(z) around the Pre-SET domain and SET domain region (Supplementary Fig. 2). RT-PCR analysis showed that EZL1 was expressed exclusively at early conjugation, while EZL2 and EZL3 were present in vegetative cells, and their expression level was strongly elevated during conjugation (Fig. 1D). $P D D 1$ expression was used as a conjugation marker and the uniform expression of HHP1 as a loading control. Knockout studies revealed that EZL1 was required for $\mathrm{H} 3 \mathrm{~K} 27 \mathrm{me} 3$ in parental macronuclei and anlagen in conjugating cells (see below), while EZL2 and EZL3 were responsible for other nonoverlapping H3K27 methylation events, including those in vegetatively growing cells (data not shown). The presence of $E(z)$ homologs in the genome of Paramecium tetraurelia (data not shown), another ciliate, provided further support for the hypothesis that PcG repression mediated by H3K27me3 originated in unicellular organisms. As ciliates likely arose earlier in evolution than fungi (Baldauf et al. 2000), the absence of E $(z)$ homologs and H3K27me3 in both Saccharomyces cerevisiae and $S$. pombe is probably due to gene loss.

\section{EZL1 encodes an H3K27 HKMT}

EZL1 mRNA was detected at a high level by RT-PCR in early conjugation and diminished as conjugation proceeded (Fig. 1D). Because its expression during conjugation appeared to overlap with the timing of key H3K27 methylation events in parental macronuclei and anlagen (Fig. 1B), EZL1 was selected for further study. Analysis of EZL1 cDNA reveals that it encodes a protein, Ezllp, of 798 amino acids (Fig. 2A). A single band of $\sim 90 \mathrm{kDa}$ was detected in cells expressing C-terminal HA-tagged EZL1 during conjugation by Western blot (Supplementary Fig. $3 \mathrm{~A}, \mathrm{~B})$, consistent with the predicted molecular weight (96 kDa). EZL1 somatic knockout strains were generated by replacing the $\mathrm{C}$-terminal half of the coding regions containing the Pre-SET and SET domains with neo3 cassette (Fig. 2A) and were confirmed by Southern blot analysis (Fig. 2B). There was no apparent phenotype in vegetatively growing and starved $\triangle E Z L 1$ cells (data not shown). RT-PCR analysis showed that EZL1 expression in conjugating $\triangle E Z L 1$ cells was completely eliminated (Fig. 2C), consistent with the exclusive expression of EZL1 from parental macronuclei during early conjugation, as is the case for TWI1 (Mochizuki et al. 2002).

We first investigated the effect of EZL1 knockout on H3K27me3 by examining the modification level throughout the conjugation by Western blot. Pdd1p was used as a conjugation-specific marker, while Hhplp and $\mathrm{H} 4$ were used as loading controls. This approach revealed that the level of pre-existing H3K27me3 in $\triangle E Z L 1$ cells gradually diminished during conjugation, while no dramatic changes were observed in wild-type cells. Importantly, H3K9me3 in late conjugation was completely abolished in $\triangle E Z L 1$ cells (Fig. 2D). Immunofluorescence staining with $\mathrm{H} 3 \mathrm{~K} 27 \mathrm{me} 3$ - and H3K9me3-specific antibodies showed that both modifications were abolished in anlagen of $\triangle E Z L 1$ cells (Fig. 2E). The specificity of the two antibodies was confirmed by peptide competition (Supplementary Fig. 1B). H3K27me3 in vegetatively growing $\triangle E Z L 1$ cells was not affected, as it was deposited by EZL2 and EZL3 (data not shown). These results were also corroborated by mass spectrometry analysis of $\triangle E Z L 1$ cells, which showed a dramatically reduced H3K27me3 level and the complete absence of methylated $\mathrm{H} 3 \mathrm{~K} 9$ in $\mathrm{H} 3$ samples from 10-h conjugating cells (Supplementary Table 1), with virtually no changes in vegetatively growing cells (data not shown). Together these data support that EZL1 encodes a conjugation-specific HKMT, with substrate specificity for H3K27 and possibly H3K9.

\section{EZL1 is required for DNA elimination and chromosome breakage in Tetrahymena}

No viable conjugation progeny were isolated from $\triangle E Z L 1$ cells (data not shown), indicating an essential function(s) of EZL1 during macronuclear development (see below). Moreover, $\triangle E Z L 1$ conjugating cells were arrested at the two micronuclei/two macronuclei stage before the readsorption of one of the two micronuclei (Supplementary Table 2), typical of other previously studied mutants that were defective in DNA elimination $(\triangle P D D 1, \triangle P D D 2, \triangle T W I 1$, and $\triangle D C L 1)$ (Coyne et al. 1999; Nikiforov et al. 1999; Mochizuki et al. 2002; Malone et al. 2005; Mochizuki and Gorovsky 2005). As mentioned above, methylated $\mathrm{H} 3 \mathrm{~K} 9$, a hallmark of DNA elimination bodies (Taverna et al. 2002), was also missing in $\triangle E Z L 1$ cells (Fig. 2D,E).

Prompted by these observations, we tested $\triangle E Z L 1$ strains for DNA elimination efficiency, which is indicative of the integrity of the heterochromatin formation pathway in anlagen. One well-studied internal eliminated sequence (IES), the M-element, was examined by single-cell PCR, using primers flanking the element. In wild-type cells, only PCR products corresponding to the two processed forms with the IES removed were detected $48 \mathrm{~h}$ into conjugation, while the unprocessed form was 
Liu et al.

A

Figure 2. $E Z L 1$ is required for $\mathrm{H} 3 \mathrm{~K} 27 \mathrm{me} 3$ and $\mathrm{H} 3 \mathrm{~K} 9 \mathrm{me} 3$ in conjugating Tetrahymena. (A) Schematic representation of endogenous EZL1 locus and the knockout construct. Full-length EZL1 mRNA is aligned with the endogenous genomic locus. In the knockout construct, $1.2 \mathrm{~kb}$ of the endogenous locus, corresponding to the conserved pre-SET and SET domain regions, was replaced by a drug-resistance cassette, neo3. The probe and restriction enzyme sites used in Southern blot analysis (shown in $B$ ) was also mapped. (X) XmnI. (B) Southern blot analysis of genomic DNA from wild-type or $\triangle E Z L 1$ cells was digested with XmnI and hybridized with the EZL1 probe. (C) RT-PCR analysis of $\triangle E Z L 1$ cells. Total RNA samples of $\triangle E Z L 1$ cells from different conjugation time points were reverse-transcribed and analyzed by PCR with primers specific for EZL1, PDD1, and HHP1. (D) Changes in $\mathrm{H} 3 \mathrm{~K} 27 \mathrm{me} 3$ and H3K9me3 levels during the conjugation pathway. Whole-cell extract samples from different conjugation time points of wild-type and $\triangle E Z L 1$ cells were resolved on $10 \%$ SDS-PAGE, blotted, and probed with the indicated antibodies. (E) Localization of H3K27me3 and H3K9me3 in conjugating cells. Wild-type and $\triangle E Z L 1$ cells from early anlagen stages were processed for immunofluorescence staining with antibodies specific for $\mathrm{H} 3 \mathrm{~K} 27 \mathrm{me} 3$, H3K9me3, or H4; nuclei were counterstained with DAPI. (Mic) Micronuclei; (AN) macronuclear anlagen; (OM) old macronuclei.

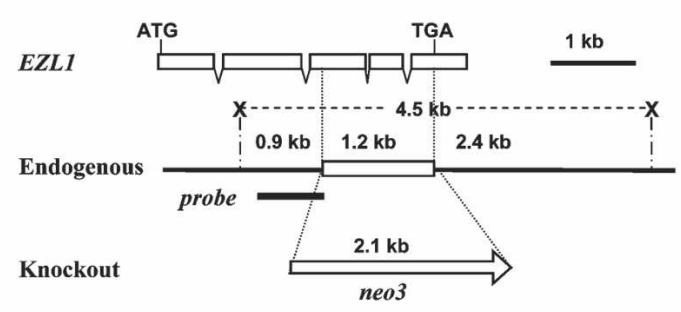

$D$

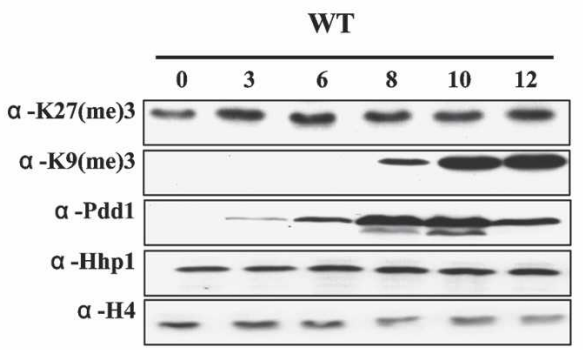

E

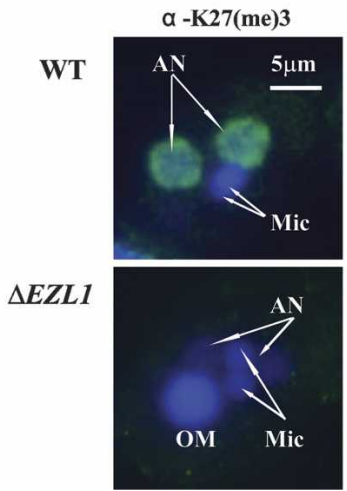

$\alpha-K 9(m e) 3$

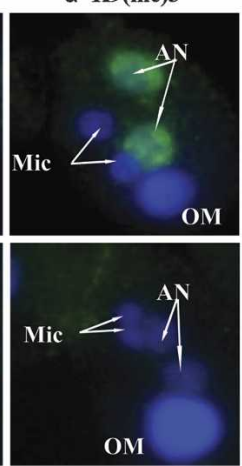

B
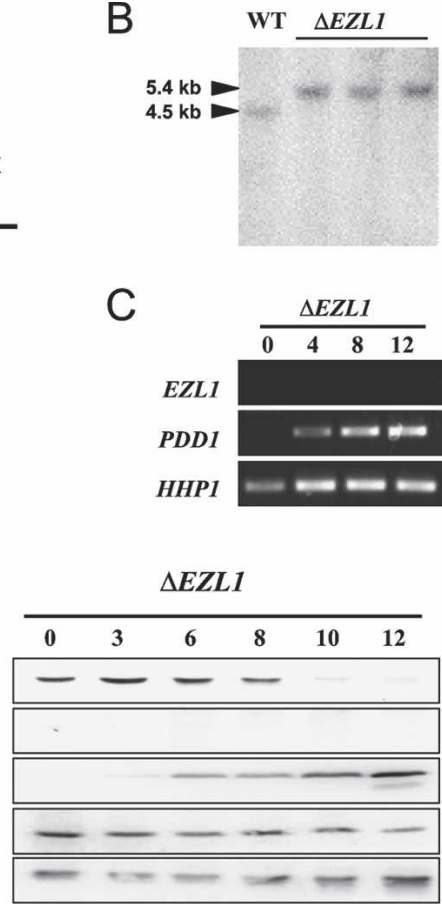

$\alpha-\mathrm{H} 4$

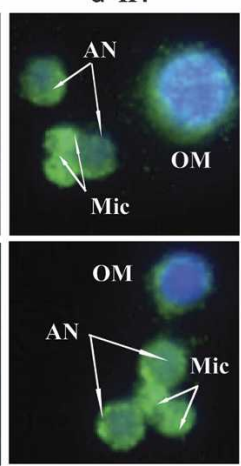

prominent in $\triangle E Z L 1$ cells (Fig. 3A). This result argues that DNA elimination is compromised in $\triangle E Z L 1$ cells. Another major genome reorganization event in anlagen is chromosome breakage, in which five micronuclear chromosomes are fragmented into hundreds of macronuclear chromosomes, followed by de novo addition of telomeres at the newly generated chromosome ends (Coyne et al. 1996). Chromosome breakage is affected in some DNA elimination-defective strains $(\triangle P D D 2$, $\triangle T W I 1$, and $\triangle D C L 1$ ) (Nikiforov et al. 1999; Mochizuki et al. 2002; Malone et al. 2005). The single-cell PCR assay was also used to examine chromosome breakage at chromosome breakage site (CBS) 819 in $\triangle E Z L 1$ cells. In wildtype conjugation progeny, only the macronuclear chromosome ends with telomeres were detected, while the micronuclear-specific form with intact CBS remained in $\triangle E Z L 1$ progeny (Fig. 3B). These results indicate that $E Z L 1$, which is required for $\mathrm{H} 3 \mathrm{~K} 27 \mathrm{me} 3$ and $\mathrm{H} 3 \mathrm{~K} 9 \mathrm{me} 3$ in anlagen, is essential for developmentally regulated het- erochromatin formation and subsequent DNA elimination and chromosome breakage.

\section{H3K27me3 physically associates with IESs}

As mentioned previously, $\mathrm{H} 3 \mathrm{~K} 27 \mathrm{me} 3$ is present in anlagen, initially in a diffuse pattern, but gradually condenses into distinct, spherical structures /with a ringlike cross-section) at the periphery of anlagen. Both the process of redistribution and the eventual configuration are similar to that of methylated H3K9 and Pdd1p, both of which mark DNA elimination bodies and physically interact with IESs (Madireddi et al. 1996; Taverna et al. 2002). Direct evidence for association between H3K27me3 and IESs was provided by chromatin immunoprecipitation (ChIP) analysis. Wild-type and $\triangle E Z L 1$ cells at 10-h conjugation were processed for ChIP and the results were quantified by real-time PCR (Fig. 3C). Four loci were analyzed: M-mic and PGM1-mic, both IES; 
A

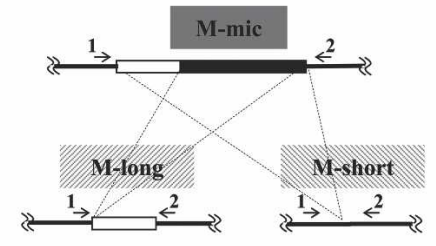

B

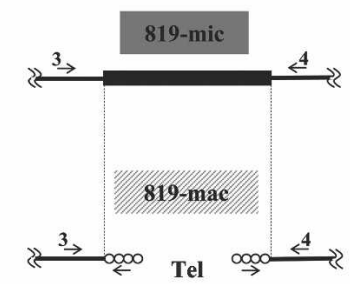

C

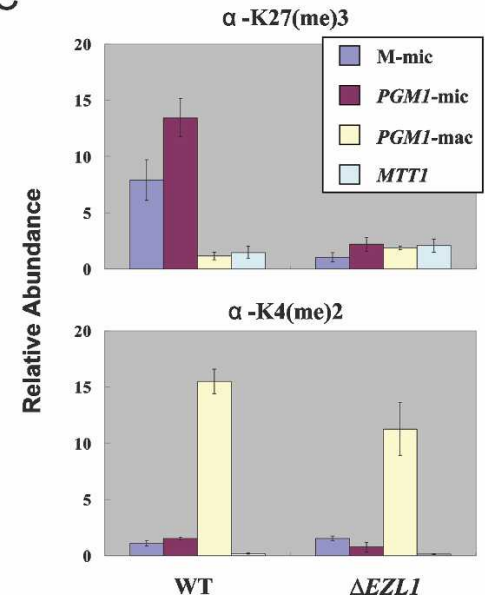

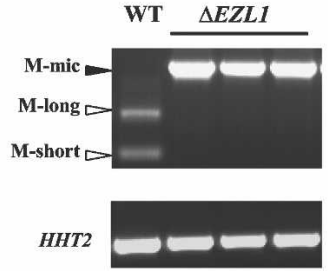
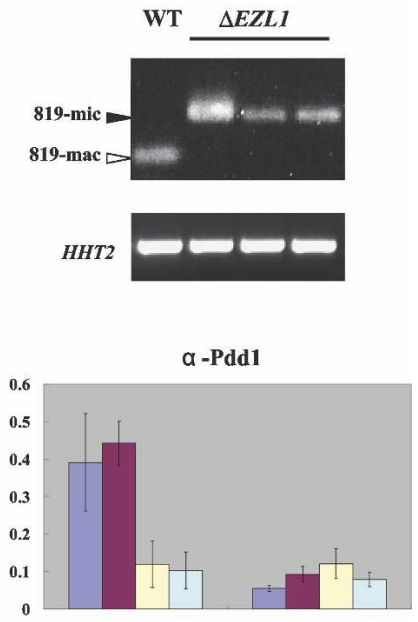

$\alpha-\mathrm{H} 3$

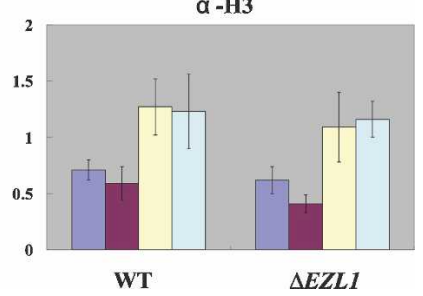

Figure 3. EZL1 is required for DNA elimination and chromosome breakage in conjugating Tetrahymena. $(A)$ Single-cell PCR assay of DNA elimination in Tetrahymena. (Left) A schematic representation of DNA elimination at M-element, an IES. Boxes indicate micronuclear-limited sequences to be eliminated; lines indicate macronuclear-retained sequences. Note that the micronuclear-specific form of M-element (M-mic) can be processed into either of the two macronuclear-specific forms (M-long and M-short). (Right) Individual conjugation progeny from wild-type and $\triangle E Z L 1$ strains were isolated at $48 \mathrm{~h}$ into conjugation and analyzed by multiplex PCR. The positions of PCR products corresponding to micronuclear- and macronuclear-specific forms are indicated by solid and open arrowheads, respectively. HHT2 locus was examined as a control for DNA integrity. $(B)$ Single-cell PCR assay of chromosome breakage in Tetrahymena. (Left) A schematic representation of CBS 819 locus. Circles indicate telomeres (Tel) added to the newly generated macronuclear chromosomal ends. (Right) PCR results from wild-type and $\triangle E Z L 1$ strains. $(C)$ Specific association of H3K27me3 with IES. Conjugating wildtype and $\triangle E Z L 1$ cells $(10 \mathrm{~h})$ were processed for ChIP with the indicated antibodies. ChIP data were quantified by real-time PCR. The results shown are from duplicate experiments and are normalized against the input (shown as percentage pulled down). See text for details.
PGM1-mac, the macronucleus-destined sequence next to PGM1-mic and a gene actively transcribed in vegetatively growing and conjugating cells; and MTT1, a gene repressed under the experimental condition. Pddlp was enriched in both IESs in wild-type cells, as expected for an IES-associated protein. Similar enrichment of IESs was observed for $\mathrm{H} 3 \mathrm{~K} 27 \mathrm{me} 3$, indicative of close associations. Strikingly, enrichment of H3K27me3 in IESs was abolished in $\triangle E Z L 1$ cells, consistent with the missing of $\mathrm{H} 3 \mathrm{~K} 27 \mathrm{me} 3$ in anlagen and its general diminishment at this stage of conjugation. Interestingly, Pddlp also failed to associate with IESs in $\triangle E Z L 1$ cells, strongly suggesting that appropriate localization of Pddlp (and, by inference, the formation of DNA elimination bodies) was dependent on H3K27me3 (and possibly H3K9me3). H3K4 dimethylation (K4me2), a euchromatin marker, was used as a negative control for IES (heterochromatin)-associated proteins. As expected, this "on" mark was found in abundance in the transcriptionally active PGM1-mac, but was absent from the transcriptionally inactive MTT1 and both IES loci in both wild-type and $\triangle E Z L 1$ cells. General H3 was used as a loading control, although it was slightly underrepresented in IES pull-down. These results support the view that $\mathrm{H} 3 \mathrm{~K} 27 \mathrm{me} 3$ plays an impor- tant role in the heterochromatin formation pathway and provides a basis for the DNA elimination defects in $\triangle E Z L 1$ cells.

\section{Ezl1p-catalyzed H3K27me3 in parental macronuclei and anlagen is RNAi dependent}

Recent studies have established that DNA elimination and the formation of the specialized heterochromatic structures in Tetrahymena are RNAi dependent, much like the pericentromeric heterochromatin formation pathway in S. pombe (Mochizuki and Gorovsky 2004b). Therefore, we sought to explore the relationship between H3K27me3 and the RNAi machinery. In knockout cells of TWI1 (Argonaute homolog) and DCL1 (Dicer homolog), both key components of the RNAi pathway required for accumulation of conjugation-specific siRNAs (Mochizuki et al. 2002; Malone et al. 2005; Mochizuki and Gorovsky 2005), as well as $\Delta E Z L 1$ cells, H3K27me3 in anlagen was greatly diminished, as shown by immunofluorescence staining (Fig. 4A). In a significant percentage of $\triangle T W I 1$ and $\triangle D C L 1$ cells $(10 \%-20 \%)$, the two new micronuclei next to the anlagen were brightly stained, while in wild-type and $\triangle E Z L 1$ cells the new mi- 
Liu et al.

A
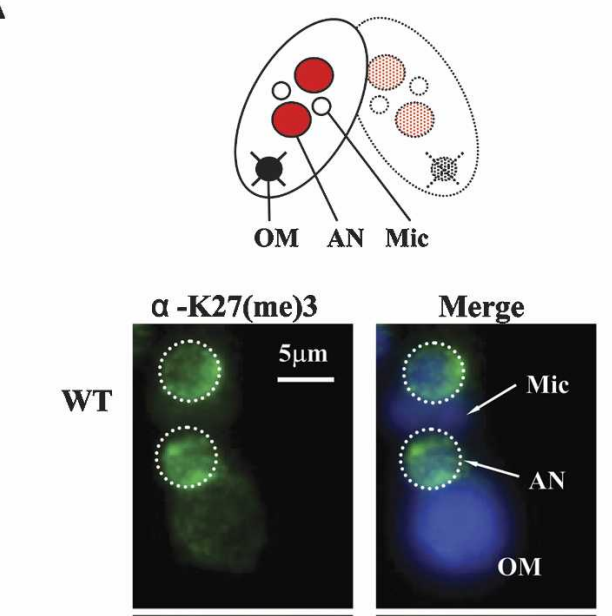

$\triangle E Z L I$
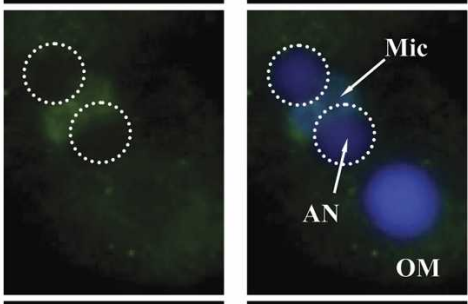

$\Delta T W I I$
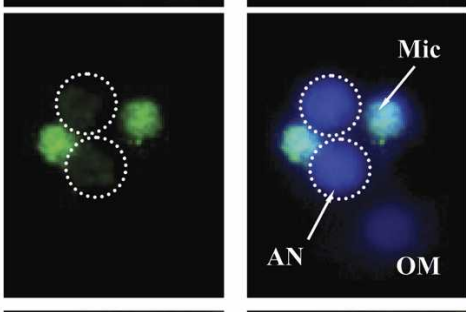

$\triangle D C L 1$
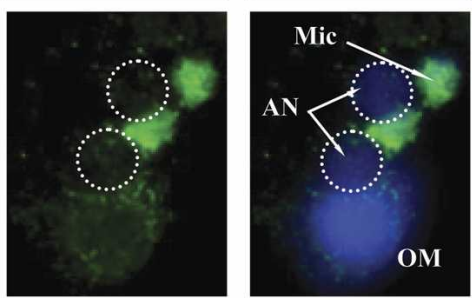

B
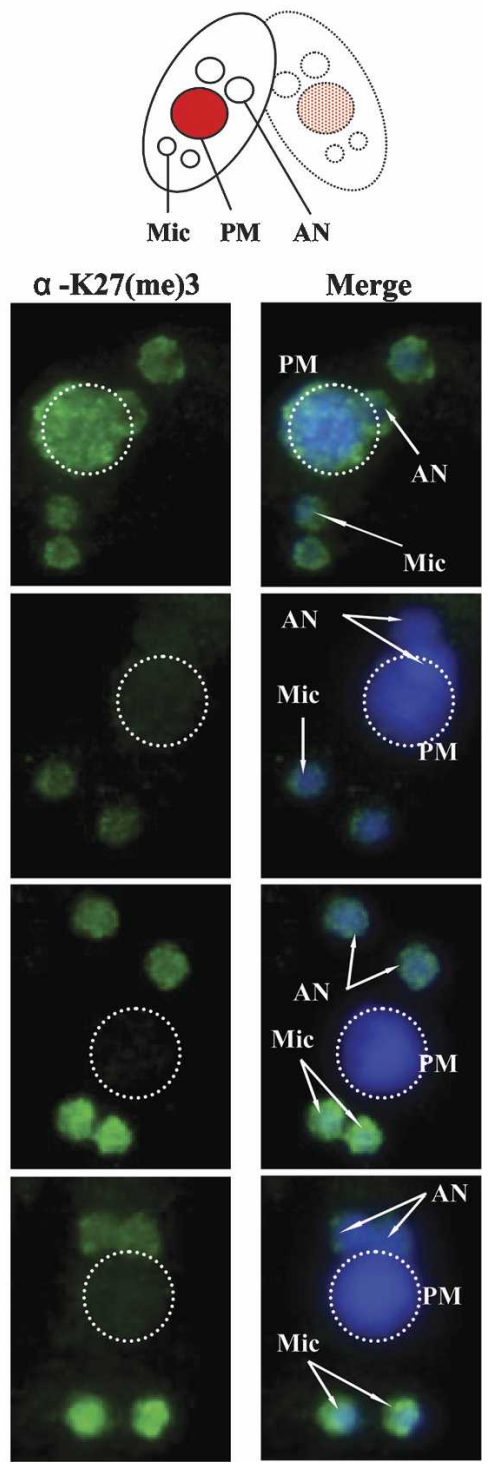

Figure 4. H3K27me3 in parental macronucleus and macronuclear anlagen in conjugating Tetrahymena is RNAi dependent. Localization of $\mathrm{H} 3 \mathrm{~K} 27 \mathrm{me} 3$ in anlagen (small white circles) at early anlagen formation (10 h conjugation) $(A)$ and in parental macronuclei (large white circles) at micronucleus/macronucleus differentiation stages (6 h conjugation) (B). Wild-type, $\triangle E Z L 1$, and RNAi-deficient $(\triangle T W I 1$ and $\triangle D C L 1)$ mutants were processed for immunofluorescence staining with H3K27me3-specific antibody and counterstained with DAPI. Note the abnormal accumulation of H3K27me3 in the new micronuclei instead of macronuclear anlagen in $\Delta T W I 1$ and $\triangle D C L 1$ cells at both stages. (Mic) Micronuclei; (PM) parental macronuclei; (AN) macronuclear anlagen; (OM) old macronuclei.

cronuclei only showed very weak signals (Fig. 4A). This may be the result of mistargeting of Ezllp or of aberrant activities of the other two $\mathrm{E}(\mathrm{z})$ homologs in Tetrahymena, which are responsible for $\mathrm{H} 3 \mathrm{~K} 27 \mathrm{me} 3$ in micronuclei in vegetatively growing and conjugating cells (data not shown). Western blot analysis of purified anlagen from wild-type, $\triangle T W I 1$, and $\triangle D C L 1$ cells at 10 -h conjugation showed greatly reduced $\mathrm{H} 3 \mathrm{~K} 27 \mathrm{me} 3$ in RNAi-deficient strains (Fig. 5A), corroborating the immunofluorescence staining results. Time-course analysis of conjugation of $\Delta T W I 1$ cells revealed that bulk H3K $27 \mathrm{me} 3$ level was diminished and H3K9me3 was abolished in late conjugation (Fig. $5 \mathrm{~B}$ ), similar to $\triangle E Z L 1$ cells. EZL1 expression was not adversely affected in $\triangle T W I 1$ cells (Fig. 5C), ruling out indirect transcriptional effects.

In wild-type cells, significant H3K27me3 was detected in parental macronuclei at early anlagen/micronuclei differentiation (Fig. 4B). However, in $\triangle E Z L 1, \Delta T W I 1$, and $\triangle D C L 1$ cells, H3K27me3 in parental macronuclei was greatly diminished (Fig. 4B). Note that H3K27me3 in the micronuclei of $\triangle E Z L 1$ was not significantly affected, while there were abnormally strong signals in the micronuclei of $\triangle T W I 1$ and $\triangle D C L 1$ cells (Fig. 4B), which apparently persisted into later anlagen formation stages 
A

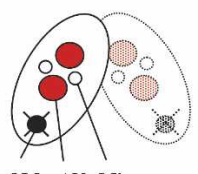

OM AN Mic

B

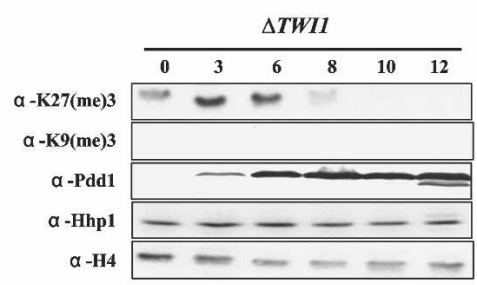

D

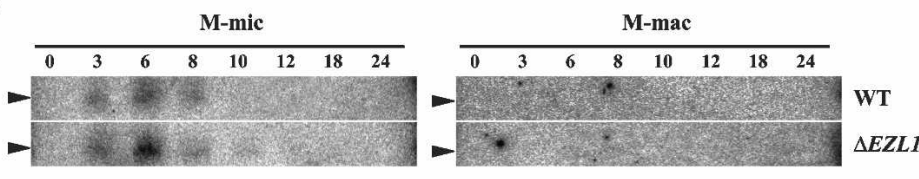

E

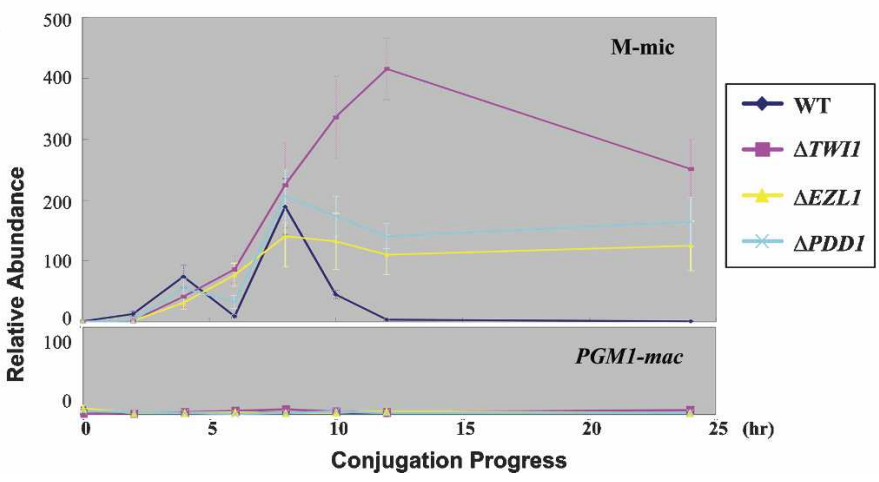

Figure 5. Relationship between H3K27 methylation and RNAi pathway. (A) Changes in H3K27me3 level in anlagen of RNAi-deficient cells. Acids extract from unit gravity-purified anlagen from 10-h conjugating wildtype, $\triangle D C L 1$, and $\triangle T W I 1$ cells were resolved on $15 \%$ SDS-PAGE, blotted, and probed with the indicated antibodies. (B) Changes in $\mathrm{H} 3 \mathrm{~K} 27 \mathrm{me} 3$ and $\mathrm{H} 3 \mathrm{~K} 9 \mathrm{me} 3$ level in $\Delta T W I 1$ cells during conjugation. Whole-cell extract samples from different conjugation time points of $\Delta$ TWI1 cells were resolved on $10 \%$ SDS-PAGE, blotted, and probed with the indicated antibodies. (C) EZL1 mRNA expression in $\triangle T W I 1$ cells. Total RNA samples from $\triangle T W I 1$ cells from different conjugation time points were reverse-transcribed and analyzed by PCR with primers specific for EZL1, PDD1, and HHP1. (D) Accumulation of M-element-specific small RNAs. Total RNA samples from wild-type and $\triangle E Z L 1$ conjugating cells were resolved by $12 \%$ sequencing gel, blotted, and probed with ${ }^{32} \mathrm{P}$ end-labeled DNA oligos specific for the micronuclear-limited sequence (M-mic) or macronuclear-retained sequence (M-mac), respectively. (E) Accumulation of IES-derived transcripts during conjugation. Total RNA samples from wild-type and DNA elimination-deficient mutants ( $\triangle T W I 1, \triangle E Z L 1$, and $\triangle P D D 1)$ at different conjugation time points $(0,2,4,6,8$, 10,12 , and $24 \mathrm{~h}$ into conjugation, respectively) were reverse-transcribed and quantified by real-time PCR with primers specific for micronuclear-limited sequence of M-element (M-mic) or the control PGM1 locus (PGM1mac). Expression levels were normalized against total RNA input (OD260) and plotted relative to the level before the initiation of conjugation $(0 \mathrm{~h})$.
(Fig. 4A). This result suggests that Ezllp-catalyzed H3K27me3 in parental macronuclei depends on the same RNAi-dependent pathway that regulates the modification in anlagen. This conclusion is further corroborated by the findings that Ezllp-HA was localized in parental macronuclei during early conjugation and anlagen at late conjugation (Supplementary Fig. 3C), as is the case for Twilp (Mochizuki et al. 2002). In wild-type cells, no dramatic changes in bulk H3K27me3 level were observed during conjugation (Fig. 2D), and immunofluorescence staining of parental macronuclei remained rather constant (Fig. 4B). However, our data suggest that this mark is apparently being actively turned over, as deficiency in H3K27 methylation leads to its dramatic loss from parental macronuclei. In wild-type cells, the H3K27me3 level in parental macronuclei drops precipitously only when they undergo degradation (old macronuclei $[\mathrm{OM}]$ ) (see Fig. 1A) during anlagen formation stages. In $\triangle E Z L 1$ and $\triangle T W I 1$ cells, diminishment of H3K27me3 in both parental macronuclei and anlagen contributes to gradual reduction of signals in the conjugation time-course analysis (Figs. 2C, 5B).

\section{Ezl1p-catalyzed H3K27me3 affects the RNAi pathway}

In S. pombe, deletion of Clr4 (the H3K9 HKMT) abolishes siRNA generation, resulting in a self-enforcing loop and interdependence of histone methylation and RNAi pathway (Volpe et al. 2002; Noma et al. 2004; Cam et al. 2005; Jia et al. 2005). In apparent contrast, the global level of siRNAs during conjugation was not significantly affected in $\triangle E Z L 1$ cells, while few siRNAs were observed in the control $\Delta$ TWI1 cells (Supplementary Fig. 4). Furthermore, we also detected similar levels of siRNAs homologous to M-element by small RNA Northern blot in $\triangle E Z L 1$ as well as wild-type cells (Fig. 5D, left panels), concentrated at early conjugation as previously reported (Chalker et al. 2005). No siRNAs homologous to the macronuclear-destined sequences of M-element were detected during conjugation (Fig. 5D, right panels). Equal loading was controlled by direct visualization of siRNAs by ethidium bromide staining (Supplementary Fig. 4). These results support that H3K27 methylation is not directly coupled with the generation of siRNAs in Tetrahymena.

We also used RT-PCR to examine the level of nongenic transcripts (Fig. 5E), precursors to conjugation-specific siRNAs. In wild-type cells, transcripts from the $\mathrm{M}$ element accumulated only in early conjugation. In RNAi-deficient $\triangle T W I 1$ cells, transcripts remained at high levels even after $24 \mathrm{~h}$ into conjugation. These results are consistent with previous characterization of wild-type and RNAi-deficient $\triangle D C L 1$ cells /Chalker and 
Yao 2001; Chalker et al. 2005; Malone et al. 2005; Mochizuki and Gorovsky 2005), demonstrating that the precursor transcripts are an integral part of the RNAi pathway. Interestingly, accumulation of M-element-specific transcripts in late conjugation was also observed in $\triangle E Z L 1$ cells and in somatic knockout cells of $P D D 1$ (Fig. $5 \mathrm{E}$ ), which encodes a chromodomain-containing protein with affinity for H3K27me3 (see below). This abnormal accumulation may be attributed to the effect of H3K27 methylation on the degradation of nongenic transcripts. Alternatively, it may be due to the failure to eliminate the IES and the resultant continuous transcription from it in these DNA elimination-deficient cells. The results hint at possible feedback interactions between H3K27 methylation and RNAi pathway.

\section{Pdd1p binds both H3K27me3 and H3K9me3}

During late conjugation stages, $\mathrm{H} 3$ in DNA elimination bodies is modified by both $\mathrm{H} 3 \mathrm{~K} 27 \mathrm{me} 3$ and $\mathrm{H} 3 \mathrm{~K} 9 \mathrm{me} 3$ (see solid arrowheads in "AN" in Fig. 1A). This "dual" methylation signature raises the question of whether these heterochromatin marks are recognized by the same or different "readers," including the two known conjugation-specific chromodomain-containing proteins, Pdd1p and Pdd3p (Madireddi et al. 1996; Smothers et al. 1997; Coyne et al. 1999; Nikiforov et al. 2000; Taverna et al. 2002). Costaining studies, using antibodies specific for the modifications and both Pddp proteins, showed that H3K27me3 and Pddlp appeared to be similarly localized, both present more at the periphery of the ringlike cross-section of DNA elimination bodies (Fig. 6A). In contrast, $\mathrm{H} 3 \mathrm{~K} 9 \mathrm{me} 3$ and Pdd3p signals were more centrally positioned within the structures (Fig. 6A). This distinct spatial distribution suggested that Pddlp may interact with $\mathrm{H} 3 \mathrm{~K} 27 \mathrm{me} 3$ while Pdd3p may bind H3K9me3. To further examine this question, we turned to fluorescence anisotropy to examine the affinity between the relevant chromodomains and the $\mathrm{H} 3$ peptides trimethylated at $\mathrm{K} 27$ or K9, respectively (Fig. 6B). Our results demonstrate that the canonical chromodomain of Pdd1p interacts strongly with both H3K27me3 and K9me3, while the chromodomain of Pdd3p preferentially recognizes H3K9me3 (Fig. 6C). Our findings are consistent with previous work showing that both Pddlp and Pdd3p are K9 methylation binders (Taverna et al. 2002). Our results also suggest an additional role for Pddlp as an effector for methylated H3K27.

In wild-type cells, Pddlp is evenly distributed in parental macronuclei at early conjugation (Coyne et al. 1999) and in anlagen, at least initially, but gradually condenses into more compacted DNA elimination bodies (Madireddi et al. 1996). This striking similarity between Pdd1p and H3K27me3 localization is most likely attributable to the direct physical interaction between them. Furthermore, mutants disrupting H3K27me3 also affected Pdd1p localization. In $\triangle D C L 1, \triangle T W I 1, \triangle E Z L 1$, and H3 K27Q mutants, Pdd1p in parental macronuclei was present as strongly stained dots (size, $<0.5 \mu \mathrm{m})$ at nuclear periphery, losing its more uniform distribution in wild-type cells (Supplementary Fig. 5). In anlagen of H3K27me3-deficient mutant cells $(\triangle D C L 1, \triangle T W I 1$, $\triangle E Z L 1)$, even at early anlagen formation stages, Pddlp was prominently present in a few large (size, $>1 \mu \mathrm{m})$, strongly stained structures (Fig. 6D), which looked like coalesced DNA elimination bodies. In both cases, the abnormal distribution is consistent with the loss of H3K27me3 and a resultant diminished affinity for Pddlp. In addition, physical association of Pddlp with IESs was compromised in $\triangle E Z L 1$ cells (Fig. 3C), underlying the abnormal localization of Pddlp in anlagen. These findings strongly support the view that Pddlp is downstream from the RNAi-dependent, Ezllp-catalyzed H3K27 methylation in Tetrahymena.

\section{Ezl1p-catalyzed H3K27 methylation regulates H3K9 methylation in anlagen}

H3K9 methylation is a previously documented specific mark for DNA elimination bodies, physically associated with IESs (Taverna et al. 2002). This mark is also downstream from the RNAi pathway and is required for DNA elimination (Liu et al. 2004). Since both methylated H3K27 and H3K9 are essential marks of the same heterochromatic structures, we were intrigued to investigate the relationship, if any, between them. Analysis by modification-specific antibodies and mass spectrometry revealed that $\mathrm{H} 3 \mathrm{~K} 9$ methylation was undetectable in anlagen of $\triangle E Z L 1$ cells (Fig. 2E; Supplementary Table 1). The result suggests that H3K27me3 in anlagen may be upstream of H3K9 methylation. To address this possibility, Tetrahymena strains with mutated histone $\mathrm{H} 3$ were generated as described previously (Liu et al. 2004). In H3 K9Q cells, which are defective in DNA elimination (Liu et al. 2004), H3K27me3 in anlagen was not significantly affected (Supplementary Fig. 6), consistent with H3K9 methylation being downstream from H3K27me3. Direct mutations at H3K27 (K27Q, K27R, and K27A) invariably led to severe meiosis defects and the mutants were arrested at early conjugation, before anlagen formation and DNA elimination (data not shown).

To circumvent this problem, a S28E strain, which did not exhibit meiosis defects, was studied, with the expectation that the glutamic acid side chain would mimic phosphorylation and interfere with methylated H3K27 binding by effectors, in a way similar to the phosphorylation-methylation "switch mechanism" regulating HP1 binding to methylated H3K9 (Fischle et al. 2003a, 2005). Indeed, fluorescence anisotropy results showed that the specific binding of Pddlp and Pdd3p were disrupted by this neighboring mutation as well as by phosphorylation (Supplementary Fig. 7). We also examined the S28E mutation for DNA elimination efficiency with an established assay for M-element processing (Duharcourt and Yao 2002; Liu et al. 2004). Only small amounts of the processed form (M-short) was detected in S28E and S10E mutant strains at $24 \mathrm{~h}$ or even $48 \mathrm{~h}$ into conjugation, while in wild-type cells M-short was predominant in late conjugation (Fig. 6E). Our results support these conclusions that the mutations disrupt the function of $\mathrm{H} 3 \mathrm{~K} 27$ and 
H3K27 and

A
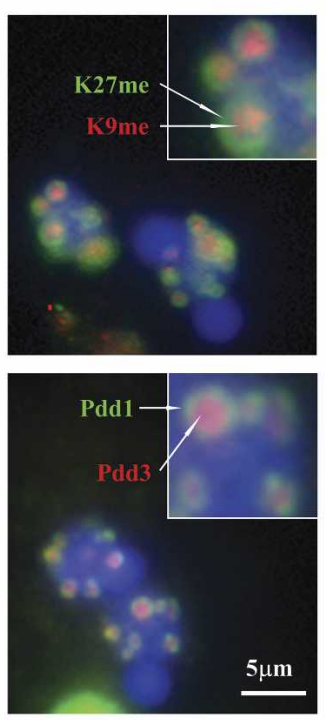

D

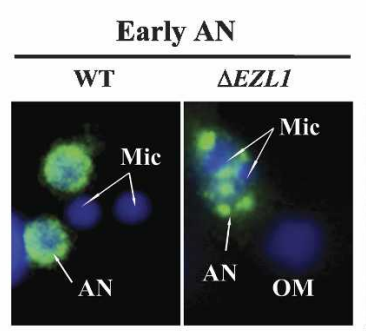

$\Delta T W I 1$

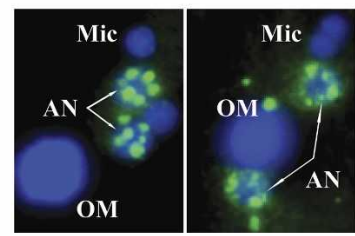

E

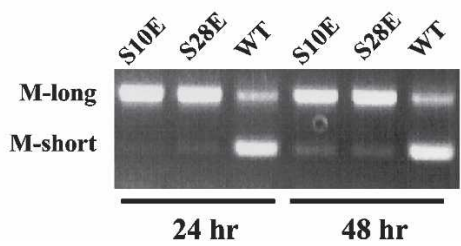

B

C

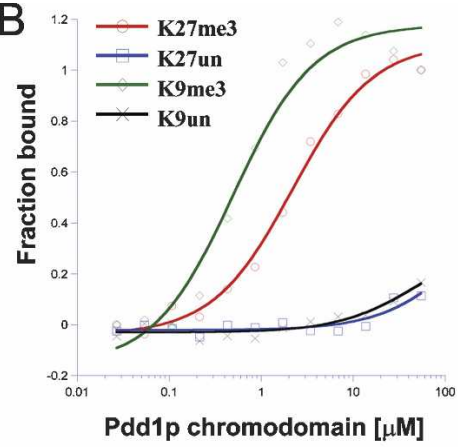

\begin{tabular}{rll} 
& \multicolumn{2}{c}{$K_{\mathrm{D}}[\mu \mathrm{M}]$} \\
\cline { 2 - 3 } Peptide & Pdd1p CD & Pdd3p CD \\
K27me3 & $2 \pm 0.5$ & $>100$ \\
K9me3 & $0.6 \pm 0.2$ & $1.0 \pm 0.3$ \\
K27un & $>100$ & $>100$ \\
K9un & $>100$ & $>100$
\end{tabular}

Late AN F

WT

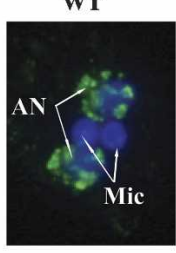

$a-K 27(m e) 3$
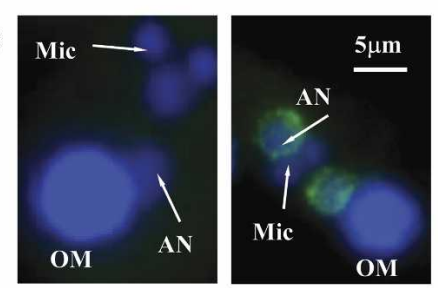

$\alpha-K 9(m e) 3$
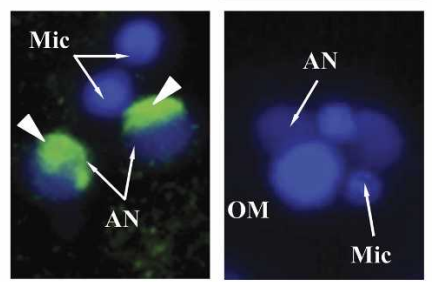

a-Pdd1
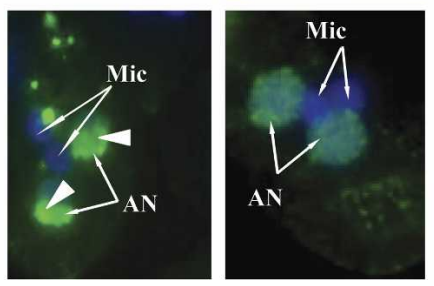

Figure 6. Pdd1p is a "dual-binder" for H3K27me3 and H3K9me3. (A) Localization of H3K27me3, H3K9me3, Pdd1p, and Pdd3p in the DNA elimination heterochromatic structures. Wild-type conjugating cells at late anlagen stages were processed for sequential doubleimmunofluorescence staining (red and green) with the indicated antibodies and counterstained with DAPI. (B) Binding specificity of Pdd1p and Pdd3p chromodomains for H3K27me3 and H3K9me3 peptides. The chromodomains (GST-tagged) were purified and incubated with the indicated FITC-labeled peptides. The interaction was quantified by fluorescence anisotropy. A representative set of binding data is shown with fitted binding curves. $(C)$ The binding affinity data $\left(\mathrm{K}_{\mathrm{D}}\right)$ calculated from triplicate experiments in $C$. $(D)$ Pdd1p localization in wild-type and H3K27me3-deficient ( $\triangle E Z L 1, \Delta T W I 1$, and $\triangle D C L 1$ ) cells at early anlagen stages (10 h). Wild-type cells at late anlagen stages are also shown. The nuclei were counterstained with DAPI. (Mic) micronuclei; (AN) macronuclear anlagen; (OM) old macronuclei. (E) PCR assay of DNA elimination efficiency. Wild-type, S10E, and S28E strains were completely assorted to have only M-long form in macronuclei. The reappearance of M-short form during conjugation can be used as an indicator for the processing efficiency of M-element (Liu et al. 2004). Mass mating cell samples were taken at $24 \mathrm{~h}$ and $48 \mathrm{~h}$ into conjugation and analyzed by PCR with primers flanking M-element. $(F)$ Immunofluorescence staining of $\mathrm{H} 3 \mathrm{~S} 10 \mathrm{E}$ and $\mathrm{H} 3 \mathrm{~S} 28 \mathrm{E}$ conjugating cells with antibodies specific for H3K27me3, H3K9me3, and Pdd1p, respectively. The nuclei were also stained with DAPI. Note the abnormal aggregation of $\mathrm{H} 3 \mathrm{~K} 9 \mathrm{me} 3$ and Pdd1p in macronuclear anlagen of S28E cells (arrowheads). 
K9 methylation, both of which are required for appropriate heterochromatin formation and DNA elimination in Tetrahymena. Immunofluorescence staining showed significant diminishment of H3K27me3 signal in S28E cells (Fig. 6F), probably due to epitope disruption by the mutation (Supplementary Fig. 8). Unexpectedly, S28E cells had dramatically increased levels of $\mathrm{H} 3 \mathrm{~K} 9 \mathrm{me} 3$ in anlagen, with the immunofluorescence signal often covering half of a nucleus (Fig. 6F). This phenotype is strikingly similar to unregulated $\mathrm{H} 3 \mathrm{~K} 9$ methylation previously reported in $\triangle D C L 1$ cells (Malone et al. 2005). In $\triangle D C L 1$ cells, while accumulation of conjugation-specific small RNA is abolished and DNA elimination fails, there is still a high level of methylated H3K9 in anlagen. However, this methyl mark is no longer enriched on IESs, indicating that the targeting mechanism is disrupted (Malone et al. 2005). Interestingly, $\triangle D C L 1$ cells also had diminished H3K27me3 in anlagen (Fig. 4B), consistent with the hypothesis that the disruption of the H3K27 methylation pathway may be the cause of unregulated H3K9 methylation. In S1OE cells, H3K9me3 signal was diminished, probably also due to epitope disruption by the mutation (Supplementary Fig. 8), with no apparent change in $\mathrm{H} 3 \mathrm{~K} 27 \mathrm{me} 3$ (Fig. 6F). Together, these results support that $\mathrm{H} 3 \mathrm{~K} 27 \mathrm{me} 3$ is upstream of $\mathrm{H} 3 \mathrm{~K} 9$ methylation and that this mark plays a significant role in regulating $\mathrm{H} 3 \mathrm{~K} 9$ methylation in anlagen, possibly by targeting it to appropriate chromatin regions by mechanisms that remain to be explored.

\section{Discussion}

RNAi-dependent heterochromatin formation mediated by PcG proteins and H3K27 methylation

Tetrahymena is one of the unicellular models in which a molecular connection between RNAi and heterochromatin formation pathways was first described (Mochizuki et al. 2002; Taverna et al. 2002) and has provided important insights into the evolutionarily conserved interactions underlying an array of epigenetic phenomena (Bernstein and Allis 2005). A link between PcG-mediated H3K27 methylation and RNAi machinery has long been suspected in higher eukaryotes, but has yet to be established. Our findings lend strong support that these two pathways are connected early in evolution. Based on our results, we propose a revised model for the DNA elimination pathway in Tetrahymena, in which H3K27 methylation plays a pivotal, and previously unrecognized, role (see Fig. 7). In brief, Ezllp, an E(z) homolog, methylates H3K27 at specific loci, targeted by conjugation-specific siRNAs. Twilp directly associates with siRNAs (Mochizuki and Gorovsky 2004a) and is required for its accumulation (Mochizuki et al. 2002). Its absence also abolishes H3K27 and H3K9 methylation in anlagen. However, the targeting mechanism remains to be defined by further studies that dissect interactions between Ezllp and RNAi machinery. In turn, H3K27 methylation modulates H3K9 methylation (see below). These modi-

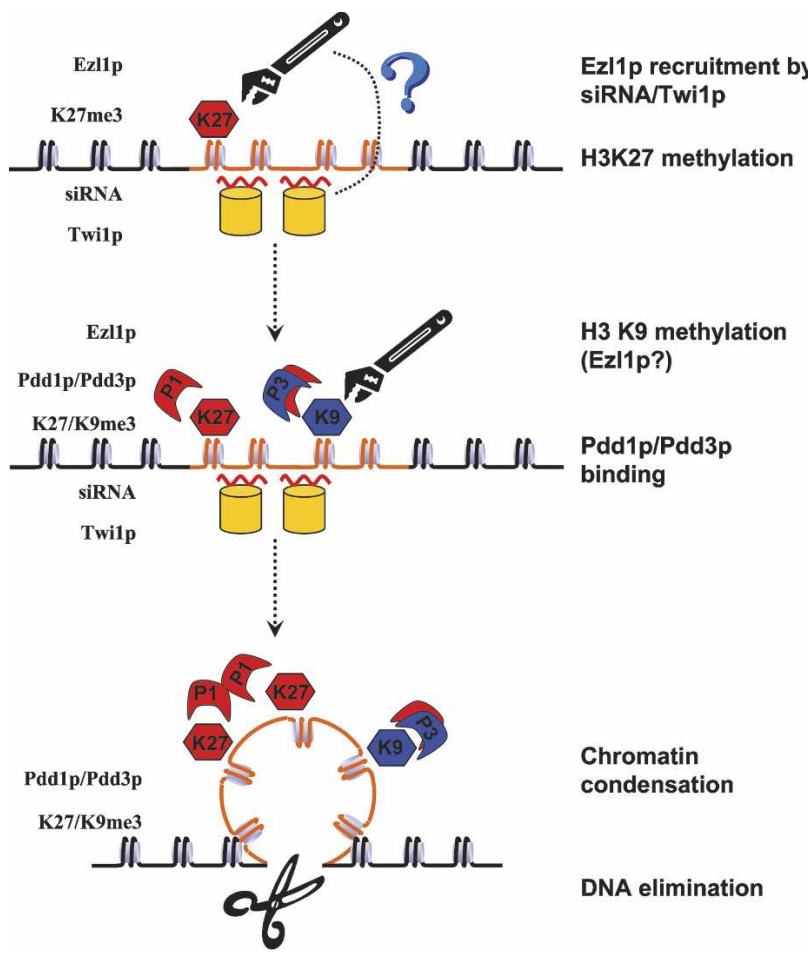

Figure 7. Revised model of RNAi-dependent heterochromatin formation pathway in Tetrahymena. Ezllp is recruited to IES regions by homologous siRNAs in association with Twilp, leading to H3K27 methylation. This is followed by H3K9 methylation, possibly also deposited by Ezllp. H3K27me3 and H3K9me3 recruit Pdd1p and Pdd3p. Chromatin condensation ensues, and eventually IES is eliminated from the macronuclear genome. For details, see text and previous proposals (Mochizuki et al. 2002; Mochizuki and Gorovsky 2004b) from which this model is derived.

fications recruit chromodomain-containing proteins, Pddlp and Pdd3p. Subsequent chromatin condensation, possibly mediated by interactions between the noncanonical chromodomains in Pddlp, leads to the formation of the specialized heterochromatic structures and eventually DNA elimination.

\section{Relationship between H3K27 methylation and H3K9 methylation}

Our results suggest that, at least in Tetrahymena, H3K9 and H3K27 methylation in anlagen coexist in the same RNAi-dependent heterochromatin formation pathway. It is possible that H3K9 as well as H3K27 methylation is catalyzed by Ezllp in Tetrahymena, as no Drosophila $\mathrm{Su}(\mathrm{var} 39$ or G9A (H3K9-specific HKMTs) homologs have yet been identified in Tetrahymena, and Drosophila $\mathrm{E}(\mathrm{z})$ has been previously demonstrated to have the dual substrate specificity (Czermin et al. 2002). In $\triangle E Z L 1$ and $\triangle T W I 1$ cells, both H3K27me3 and H3K9me3 are abolished in anlagen. However, in S28E as well as $\triangle D C L 1$ cells, which abrogate $\mathrm{H} 3 \mathrm{~K} 27 \mathrm{me} 3, \mathrm{H} 3 \mathrm{~K} 9 \mathrm{me} 3$ is abnormally increased and apparently mistargeted. One 
explanation for the results is that H3K27 methylation may demarcate the boundary between IES and macronuclear-destined sequences, and thus limit the extent of H3K9 methylation. Alternatively, H3K27 methylation may help to target H3K9 methylation to IES.

Cross-talk between H3K9 and H3K27 methylation has been documented in higher eukaryotes. In Drosophila, a function of H3K27 methylation in heterochromatic gene silencing is indicated by the enhancement of positioneffect variegation (PEV) after E(z) overexpression (Laible et al. 1997), as well as diminished PEV in E(z) and Su(z)12 [an E(z)-associated protein essential for H3K27 HKMT activities] loss-of-function mutants (Birve et al. 2001). In Ezh2 [a mammalian E(z) homolog]-null parthenogenetic mouse embryonic cells, H3K9me3 in pericentromeric regions, as well as global K27 methylation is greatly diminished (Erhardt et al. 2003). Conversely, in Suv39h1/ Suv39h2 (H3K9 HKMTs) double-null mouse ES cells, both $\mathrm{H} 3 \mathrm{~K} 9 \mathrm{me} 2$ and $\mathrm{H} 3 \mathrm{~K} 9 \mathrm{me} 3$ in pericentromeric regions are abolished, while $\mathrm{H} 3 \mathrm{~K} 27 \mathrm{me} 3$ accumulates there (Peters et al. 2003), coincident with an abnormally strong localization of Ezh2 (T. Jenuwein, pers. comm.). Furthermore, Pdd1p has affinity for both H3K27me3 and H3K9me3. The presence of Polycomb homologs with similar dual binding capabilities in mammals (Bernstein et al. 2006) and a chromodomain-containing DNA methyltransferase whose binding and chromatin recruitment require methylated $\mathrm{H} 3 \mathrm{~K} 9$ and $\mathrm{H} 3 \mathrm{~K} 27$ in plants (Lindroth et al. 2004) also supports that overlap between the functions of the two modifications may be widespread.

The role of Pdd1p, a chromodomain protein involved in programmed DNA elimination

Our work indicates that Pddlp is a conjugation-specific chromodomain protein that can bind H3K27me3 as well as H3K9me3. Previous studies have shown that it decorates DNA elimination bodies (Madireddi et al. 1994, 1996) and is required for DNA elimination (Coyne et al. 1999). It has also been suggested to play a role in coupling the RNAi and heterochromatin formation pathway (Mochizuki et al. 2002). In S. pombe, two chromodomain proteins are known to be involved in RNAi-dependent heterochromatin formation, Chp1 and Swi6; both are methylated H3K9 binders (Hall et al. 2002; Partridge et al. 2002). In parallel, Pdd1p may play a role similar to Chp1, an integral component of the RNAi-induced transcriptional silencing (RITS) complex that couples the H3K9 methylation and RNAi pathway (Verdel et al. 2004). However, a direct interaction between Pdd1p and Twilp (analogous to Chpl and Agol in RITS complex) has yet to be established in Tetrahymena. In addition, our studies show that accumulation of siRNA is not affected in $\triangle P D D 1$ cells (Supplementary Fig. 4), in apparent contrast to a previous report (Mochizuki et al. 2002), providing genetic evidence that $P D D 1$ may not function upstream of siRNA generation. Alternatively, Pdd1p may act like Swi6/HP1 in S. pombe/Drosophila, stabilizing heterochromatin structure and reinforcing TGS. In fact, direct tethering of Pddlp to a sequence normally destined for macronuclear genome leads to its elimination instead (Taverna et al. 2002). We conclude that Pddlp as a downstream effector of histone methylation is currently most consistent with the available evidence, which is integrated into our model for the heterochromatin formation pathway in Tetrahymena (Fig. 7). However, this does not completely rule out certain feedback effects that Pddlp may have on RNAi and histone methylation events. There is abnormal accumulation of nongenic transcripts at late conjugation in $\triangle P D D 1$ cells, similar to what is observed in RNAi deficient mutants like $\triangle D C L 1$ and $\triangle T W I 1$. In S. pombe, deletion of Swi6 also leads to accumulation of precursor transcripts, as TGS is compromised (Noma et al. 2004). Additionally, it is possible that Pddlp may mediate the transition between H3K27 and H3K9 methylation. In support, H3K9 methylation in anlagen is significantly reduced in $\triangle P D D 1$ cells (Taverna et al. 2002), but H3K27 methylation is not affected (data not shown). However, the interpretation is complicated by zygotic expression of PDD1 in the somatic knockout cells, which at least partially restores the Pdd1p level during the anlagen formation stages. Further studies of PDD1 germline/somatic knockout homokaryons should help to resolve this issue.

Interactions between H3K27 methylation and RNAi pathways

In $S$. pombe, H3K9 methylation and the RNAi pathway are coupled through the RITS complex (Verdel et al. 2004). Indeed, chromatin association of RITS is essential for efficient processing of RNA transcripts and generation of siRNAs, as both the deletion of Clr4 (H3K9 HKMT) and the mutation of Chp1 (abolishing binding of methylated H3K9) lead to elimination of siRNAs (Noma et al. 2004). This coupling is apparently absent in Tetrahymena, as accumulation of siRNAs is not negatively affected in $\triangle E Z L 1$ cells, even though there is abnormal accumulation of the precursor RNA transcripts. The tight coupling in $S$. pombe forms a self-enforcing loop and helps to propagate the heterochromatin state, while in Tetrahymena the developmentally regulated heterochromatin formation is followed by DNA elimination, making the maintenance mechanism dispensable. Generation of siRNAs and their targeting to chromatin regions are most likely separated physically and mechanistically in the latter scenario. As post-TGS (PTGS) and TGS coexist in several systems (Garnier et al. 2004; Matzke and Birchler 2005; Howard-Till and Yao 2006), the utilization of ectopically generated siRNAs to target histone modifications to genomic loci may be a common theme.

\section{Materials and methods}

Strains and culture conditions

Wild-type Tetrahymena thermophila strains CU427 and CU428 (provided by Dr. Donna Cassidy-Hanley, Tetrahymena 
Stock Center, Cornell University, Ithaca, NY) were grown in SPP medium at $30^{\circ} \mathrm{C}$. Conjugation was initiated when log-phase cells of different mating types were washed, starved (16-24 h at $\left.30^{\circ} \mathrm{C}\right)$, and mixed in $10 \mathrm{mM}$ Tris buffer $(\mathrm{pH} 7.5)$ or Dryl's phosphate buffer $\left(1.5 \mathrm{mM} \mathrm{CaCl}_{2}, 1 \mathrm{mM} \mathrm{NaH}_{2} \mathrm{PO}_{4}, 1 \mathrm{mM} \mathrm{Na}_{2} \mathrm{HPO}_{4}\right.$, $2 \mathrm{mM}$ sodium citrate)

\section{Identification and characterization of EZL1}

EZLS were identified by their homolog to Drosophila $\mathrm{E}(\mathrm{z})$ at its SET and pre-SET domain in Tetrahymena macronuclear genome (described in Eisen et al. 2006; for BLAST search, http:// seq.ciliate.org/cgi-bin/blast-tgd.pl). Part of their cDNAs encoding the conserved Pre-SET and SET domain was confirmed by sequencing. Full-length cDNA of EZL1 is compiled from data generated by conjugating Tetrahymena cDNA library sequencing project (accession no. EF446990).

\section{Generation of EZL1 knockout and HA-tagging strains}

To generate the EZL1 knockout construct, its genomic DNA (CH445625:206853..211508 of Tetrahymena thermophila SB210 scf_8254610 genomic scaffold) was amplified and cloned into pBlueScript $\mathrm{SK}(+)$ vector. The internal fragment (CH445625:208453..209634) was replaced with the neo3 cassette, which confers paromomycin resistance in Tetrahymena cells (Shang et al. 2002). For C-terminal HA-tagging of EZL1, tandem Flag-HA tag (as described in Tagami et al. 2004) was inserted before the stop codon of EZL1 by overlapping PCR. The neo3 cassette was inserted into 3' untranslated region (UTR), $\sim 500$ base pairs (bp) downstream from the stop codon (CH445625:210126) at an engineered SmaI site. CU427 and CU428 were mated, and the constructs were introduced by standard biolistic transformations (Cassidy-Hanley et al. 1997). Transformants were selected by paromomycin resistance, and complete replacement was confirmed by Southern blot analysis.

\section{Indirect immunofluorescence staining}

Tetrahymena cells were fixed in partial Schaudin's fixative (Madireddi et al. 1994) or $2 \%$ paraformaldehyde (Smothers et al. 1997), as previously described. The primary antibodies used were $\alpha$-H3K27me3 (1:500; Millipore, 07-449), $\alpha$-H3K9me3 (1:100; Millipore, 07-442), $\alpha$-Pdd1p (1:100; Abcam, ab5338), and $\alpha$-Pdd3p (1:100; Abcam, ab5340). The secondary antibodies used were Alexa Fluor 488-conjugated goat anti-rabbit IgG (1:1000; Invitrogen, A-11008) or Cy3-conjugated second secondary antibody (1:1000; Jackson ImmunoResearch, 111-165-144). Digital images were collected using SPOT software on a Zeiss Axioskop 2 Plus microscope.

For dual staining of $\mathrm{H} 3 \mathrm{~K} 9 \mathrm{me} 3 / \mathrm{K} 27 \mathrm{me} 3$ or $\mathrm{Pdd} 3 / \mathrm{Pdd} 1$, cells were fixed in $2 \%$ paraformaldehyde and prepared as described previously (Smothers et al. 1997) with the following modifications. After incubation in the first primary antibody overnight (either $\alpha$-H3K27me3 [1:500] or $\alpha$-Pdd1 [1:100; Abcam, ab5338]), cells were washed five times in PHEM wash buffer and incubated with Alexa Fluor 488-conjugated goat anti-rabbit IgG (1:700; Invitrogen, A-11008) as the first secondary antibody for 1 h. Following five washes with PHEM wash buffer, cells were incubated with AffiniPure Fragment Goat Anti-Rabbit IgG $(\mathrm{H}+\mathrm{L})$ (1:70; Jackson ImmunoResearch, 111-007-003) for $1 \mathrm{~h}$ to block the first secondary antibody. After five washes with PHEM wash buffer, cells were incubated in the second primary antibody ( $\alpha$-H3K9me3 [1:50] or $\alpha$-Pdd3 [1:100; Abcam, ab5340]) for $1 \mathrm{~h}$ and washed five times in PHEM wash buffer. Cells were then incubated with Cy3-conjugated second secondary antibody
(1:800; Jackson ImmunoResearch, 111-165-144) for $1 \mathrm{~h}$, washed five times with PHEM wash buffer, and stained with DAPI (1 $\mu \mathrm{g} / \mathrm{mL}$; Roche Diagnostics). The $\alpha$-H3K9me3 and $\alpha$-H3K27me3 antibodies were a gracious gift of T. Jenuwein (Institute of Molecular Pathology, Vienna, Austria).

PCR analysis of DNA elimination and chromosome breakage

Single conjugation progeny was isolated and prepared as previously described (Coyne et al. 1999; Mochizuki et al. 2002). The cell lysate was analyzed by two successive rounds of PCR (30 cycles each) with nested primers. The following primers were used for analysis of DNA elimination at M-element and chromosome breakage at CBS819: M5'-1, M5'-2, M3'-1, M3'-2; CBS5'-1, CBS5'-2, CBS3'-1, CBS3'-2, and the telomere primer (see the Supplemental Material for primer sequences). The two macronuclear forms of CBS819 are of very similar size ( 140 bp) and appear as a single band in an agarose gel. The accession numbers of M-element and CBS819 are M21936 and M15711, respectively.

\section{$R T-P C R$}

Total RNA was extracted with Trizol reagent (Invitrogen) and treated with amplification grade DNase I (Invitrogen). First strand cDNA was synthesized using SuperScript III reverse transcriptase (Invitrogen) and random hexamer primers (New England Biolabs). It was then followed by either conventional PCR amplification or quantitative-PCR analysis. See the Supplemental Material for details.

\section{Observation of small RNAs}

Total RNA was extracted with Trizol reagent (Invitrogen) and dissolved in formamide. RNA from $\sim 5 \times 10^{4}$ cells was separated on $12 \%$ polyacrylamide-urea DNA sequencing gels (Sequagel, National Diagonostics) and visualized by soaking the gels in 1.5 $\mu \mathrm{g} / \mathrm{mL}$ ethidium bromide (Mochizuki et al. 2002). Small RNAs can also be electro-transferred ( $1 \times \mathrm{TBE}, 200 \mathrm{~V}, 45 \mathrm{~min})$ to highbond- $\mathrm{N}^{+}$membrane (Amersham Biosciences) for Northern blot analysis as described (Howard-Till and Yao 2006). See the Supplemental Material for probe sequences.

\section{ChIP}

Cells were cross-linked, harvested, and washed as described previously (Dedon et al. 1991; Taverna et al. 2002). Cells were resuspended at $5 \times 10^{4}$ cells $/ \mathrm{mL}$ in SDS lysis buffer (Chadee et al. 1999), and sonication was performed in Bioruptor (Diagenode; high-power output, 0.5 -min pulse/0.5-min interval cycle, $8 \mathrm{~min}$ total). Cell lysates were immunoprecipitated with $\alpha$-H3K27me3 (Millipore, 07-449), $\alpha$-H3K4me2 (Abcam, ab7766), $\alpha$-Pdd1p (Abcam, ab5338), and $\alpha$-H3 general (laboratory generated), respectively. Immunoprecipitation and washing of the protein A-bound chromatin were done as previously described (Boggs et al. 2002). Quantitative-PCR was performed using SYBR green PCR master mix (Applied Biosystems) and MX3000P real-time PCR machine (Strategene). See the Supplemental Material for primer sequences.

\section{Fluorescence anisotropy analysis}

The first chromodomain (the only canonical chromodomain with the three "caging" aromatic residues) of Pddlp and the only chromodomain of Pdd3p were codon-corrected (Taverna et al. 2002) and subcloned into a GST-tagging vector (pGEX4T2, Strategene). Concentration of the purified proteins was deter- 
mined by absorbance spectrometry using predicted extinction coefficients (for GST-Pdd1p CD1, $\varepsilon_{280}=61,590 \mathrm{M}^{-1} \mathrm{~cm}^{-1}$; for GST-Pdd3p CD1, $\varepsilon_{280}=62,870 \mathrm{M}^{-1} \mathrm{~cm}^{-1}$ ). FITC-labeled peptide concentrations were determined by absorbance of the chromogroup $\left(\varepsilon_{492}=68,000 \mathrm{M}^{-1} \mathrm{~cm}^{-1}\right)$. Binding assays were performed in $20 \mathrm{mM}$ sodium phosphate buffer containing $25 \mathrm{mM} \mathrm{NaCl}, 2$ $\mathrm{mM}$ DTT, and $0.1 \%$ Tween-20 in the presence of $120 \mathrm{nM}$ FITClabeled peptide. Data were obtained with a Hidex chameleon plate reader. Data analysis and curve fitting were done by Kaleidagraph software.

\section{Mass spectrometry analysis}

RP-HPLC-purified H3 was treated with propionylation reagent, digested with trypsin, and treated with propionylation reagent again after digestion (Syka et al. 2004). Peptide mixtures were gradient-eluted into a linear quadrupole ion trap Fourier-transform ion cyclotron resonance mass spectrometer (Finnigan LTQ-FTMS, Thermo Electron Corp.) equipped with a modified microelectrospray ionization source ( $\mu E S I)$ (Martin et al. 2000). For comparative analysis of histone post-translational modifications, stable isotope labeling of the peptide carboxylic groups was employed (Syka et al. 2004). For details, see the Supplemental Material.

\section{Acknowledgments}

$\triangle D C L 1$ strains were kindly provided by Douglas L. Chalker. $\Delta$ TWI1 strains were kindly provided by Martin A. Gorovsky and Kazufumi Mochizuki, with whom we also had fruitful discussions about the studies. The H3K27me3-specific antibody initially used was a gift from Thomas Jenuwein. We are indebted to Emily Bernstein for technical assistance in the characterization of siRNA in Tetrahymena, and critical input in the development of Pdd1p and Pdd3p antibodies. We appreciate the critical reading of the manuscript by Martin A. Gorovsky, Kathleen Collins, and Monika Lachner. This work was supported by the Rockefeller University and grants from the National Institutes of Health (R01 GM63959 to C.D.A. and R01 GM37537 to D.F.H.). Y.L. was supported by a Ruth L. Kirschstein National Research Service Award (GM075575) from the National Institutes of Health. Preliminary sequence data of Tetrahymena macronuclear genome were obtained from the Institute for Genomic Research (http://www.tigr.org).

\section{References}

Allis, C.D., Bowen, J.K., Abraham, G.N., Glover, C.V., and Gorovsky, M.A. 1980. Proteolytic processing of histone H3 in chromatin: A physiologically regulated event in Tetrahymena micronuclei. Cell 20: 55-64.

Baldauf, S.L., Roger, A.J., Wenk-Siefert, I., and Doolittle, W.F. 2000. A kingdom-level phylogeny of eukaryotes based on combined protein data. Science 290: 972-977.

Bannister, A.J., Zegerman, P., Partridge, J.F., Miska, E.A., Thomas, J.O., Allshire, R.C., and Kouzarides, T. 2001. Selective recognition of methylated lysine 9 on histone $\mathrm{H} 3$ by the HP1 chromo domain. Nature 410: 120-124.

Bastow, R., Mylne, J.S., Lister, C., Lippman, Z., Martienssen, R.A., and Dean, C. 2004. Vernalization requires epigenetic silencing of FLC by histone methylation. Nature 427: 164167.

Bender, L.B., Cao, R., Zhang, Y., and Strome, S. 2004. The MES2/MES-3/MES-6 complex and regulation of histone H3 methylation in C. elegans. Curr. Biol. 14: 1639-1643.

Bernstein, E. and Allis, C.D. 2005. RNA meets chromatin. Genes \& Dev. 19: 1635-1655.
Bernstein, E., Duncan, E.M., Masui, O., Gil, J., Heard, E., and Allis, C.D. 2006. Mouse polycomb proteins bind differentially to methylated histone $\mathrm{H} 3$ and RNA and are enriched in facultative heterochromatin. Mol. Cell. Biol. 26: 2560-2569.

Birve, A., Sengupta, A.K., Beuchle, D., Larsson, J., Kennison, J.A., Rasmuson-Lestander, A., and Muller, J. 2001. Su(z)12, a novel Drosophila Polycomb group gene that is conserved in vertebrates and plants. Development 128: 3371-3379.

Boggs, B.A., Cheung, P., Heard, E., Spector, D.L., Chinault, A.C., and Allis, C.D. 2002. Differentially methylated forms of histone $\mathrm{H} 3$ show unique association patterns with inactive human X chromosomes. Nat. Genet. 30: 73-76.

Cam, H.P., Sugiyama, T., Chen, E.S., Chen, X., FitzGerald, P.C., and Grewal, S.I. 2005. Comprehensive analysis of heterochromatin- and RNAi-mediated epigenetic control of the fission yeast genome. Nat. Genet. 37: 809-819.

Cao, R., Wang, L., Wang, H., Xia, L., Erdjument-Bromage, H., Tempst, P., Jones, R.S., and Zhang, Y. 2002. Role of histone H3 lysine 27 methylation in Polycomb-group silencing. Science 298: 1039-1043.

Cassidy-Hanley, D., Bowen, J., Lee, J.H., Cole, E., VerPlank, L.A., Gaertig, J., Gorovsky, M.A., and Bruns, P.J. 1997. Germline and somatic transformation of mating Tetrahymena thermophila by particle bombardment. Genetics 146: $135-147$.

Chadee, D.N., Hendzel, M.J., Tylipski, C.P., Allis, C.D., BazettJones, D.P., Wright, J.A., and Davie, J.R. 1999. Increased Ser10 phosphorylation of histone $\mathrm{H} 3$ in mitogen-stimulated and oncogene-transformed mouse fibroblasts. J. Biol. Chem. 274: 24914-24920.

Chalker, D.L. and Yao, M.C. 2001. Nongenic, bidirectional transcription precedes and may promote developmental DNA deletion in Tetrahymena thermophila. Genes \& Dev. 15: 1287-1298.

Chalker, D.L., Fuller, P., and Yao, M.C. 2005. Communication between parental and developing genomes during Tetrahymena nuclear differentiation is likely mediated by homologous RNAs. Genetics 169: 149-160.

Coyne, R.S., Chalker, D.L., and Yao, M.C. 1996. Genome downsizing during ciliate development: Nuclear division of labor through chromosome restructuring. Annu. Rev. Genet. 30: 557-578.

Coyne, R.S., Nikiforov, M.A., Smothers, J.F., Allis, C.D., and Yao, M.C. 1999. Parental expression of the chromodomain protein Pddlp is required for completion of programmed DNA elimination and nuclear differentiation. Mol. Cell 4: 865-872.

Czermin, B., Melfi, R., McCabe, D., Seitz, V., Imhof, A., and Pirrotta, V. 2002. Drosophila enhancer of Zeste/ESC complexes have a histone $\mathrm{H} 3$ methyltransferase activity that marks chromosomal Polycomb sites. Cell 111: 185-196.

Dedon, P.C., Soults, J.A., Allis, C.D., and Gorovsky, M.A. 1991. Formaldehyde cross-linking and immunoprecipitation demonstrate developmental changes in $\mathrm{H} 1$ association with transcriptionally active genes. Mol. Cell. Biol. 11: 1729-1733.

Duharcourt, S. and Yao, M.C. 2002. Role of histone deacetylation in developmentally programmed DNA rearrangements in Tetrahymena thermophila. Eukaryot. Cell 1: 293-303.

Eisen, J.A., Coyne, R.S., Wu, M., Wu, D., Thiagarajan, M., Wortman, J.R., Badger, J.H., Ren, Q., Amedeo, P., Jones, K.M., et al. 2006. Macronuclear genome sequence of the ciliate Tetrahymena thermophila, a model eukaryote. PLOS Biol. doi: 10.1371/journal.pbio.0040286.

Erhardt, S., Su, I.H., Schneider, R., Barton, S., Bannister, A.J., Perez-Burgos, L., Jenuwein, T., Kouzarides, T., Tarakhovsky, A., and Surani, M.A. 2003. Consequences of the depletion of 
zygotic and embryonic enhancer of zeste 2 during preimplantation mouse development. Development 130: 4235-4248.

Fischle, W., Wang, Y., and Allis, C.D. 2003a. Binary switches and modification cassettes in histone biology and beyond. Nature 425: 475-479.

Fischle, W., Wang, Y., Jacobs, S.A., Kim, Y., Allis, C.D., and Khorasanizadeh, S. 2003b. Molecular basis for the discrimination of repressive methyl-lysine marks in histone $\mathrm{H} 3$ by Polycomb and HP1 chromodomains. Genes \& Dev. 17: 1870-1881.

Fischle, W., Tseng, B.S., Dormann, H.L., Ueberheide, B.M., Garcia, B.A., Shabanowitz, J., Hunt, D.F., Funabiki, H., and Allis, C.D. 2005. Regulation of HP1-chromatin binding by histone H3 methylation and phosphorylation. Nature 438: $1116-1122$

Garcia, B.A., Hake, S.B., Diaz, R.L., Kauer, M., Morris, S.A., Recht, J., Shabanowitz, J., Mishra, N., Strahl, B.D., Allis, C.D., et al. 2007. Organismal differences in post-translational modifications in histones $\mathrm{H} 3$ and H4. J. Biol. Chem. 282: 7641-7655.

Garnier, O., Serrano, V., Duharcourt, S., and Meyer, E. 2004. RNA-mediated programming of developmental genome rearrangements in Paramecium tetraurelia. Mol. Cell. Biol. 24: 7370-7379.

Grewal, S.I. and Moazed, D. 2003. Heterochromatin and epigenetic control of gene expression. Science 301: 798-802.

Grimaud, C., Bantignies, F., Pal-Bhadra, M., Ghana, P., Bhadra, U., and Cavalli, G. 2006. RNAi components are required for nuclear clustering of Polycomb group response elements. Cell 124: 957-971.

Hall, I.M., Shankaranarayana, G.D., Noma, K., Ayoub, N., Cohen, A., and Grewal, S.I. 2002. Establishment and maintenance of a heterochromatin domain. Science 297: 2232-2237.

Howard-Till, R.A. and Yao, M.C. 2006. Induction of gene silencing by hairpin RNA expression in Tetrahymena thermophila reveals a second small RNA pathway. Mol. Cell. Biol. 26: $8731-8742$.

Huang, H., Wiley, E.A., Lending, C.R., and Allis, C.D. 1998. An HP1-like protein is missing from transcriptionally silent micronuclei of Tetrahymena. Proc. Nat1. Acad. Sci. 95: 1362413629.

Huang, H., Smothers, J.F., Wiley, E.A., and Allis, C.D. 1999. A nonessential HP1-like protein affects starvation-induced assembly of condensed chromatin and gene expression in macronuclei of Tetrahymena thermophila. Mol. Cell. Biol. 19: 3624-3634.

Jahn, C.L. and Klobutcher, L.A. 2002. Genome remodeling in ciliated protozoa. Annu. Rev. Microbiol. 56: 489-520.

Jia, S., Kobayashi, R., and Grewal, S.I. 2005. Ubiquitin ligase component Cul4 associates with $\mathrm{Clr} 4$ histone methyltransferase to assemble heterochromatin. Nat. Cell Biol. 7: 10071013.

Karrer, K.M. 2000. Tetrahymena genetics: Two nuclei are better than one. Methods Cell Biol. 62: 127-186.

Kim, D.H., Villeneuve, L.M., Morris, K.V., and Rossi, J.J. 2006. Argonaute-1 directs siRNA-mediated transcriptional gene silencing in human cells. Nat. Struct. Mol. Biol. 13: 793-797.

Kuzmichev, A., Nishioka, K., Erdjument-Bromage, H., Tempst, P., and Reinberg, D. 2002. Histone methyltransferase activity associated with a human multiprotein complex containing the Enhancer of Zeste protein. Genes \& Dev. 16: 2893 2905.

Lachner, M., O'Carroll, D., Rea, S., Mechtler, K., and Jenuwein, T. 2001. Methylation of histone H3 lysine 9 creates a binding site for HP1 proteins. Nature 410: 116-120.
Laible, G., Wolf, A., Dorn, R., Reuter, G., Nislow, C., Lebersorger, A., Popkin, D., Pillus, L., and Jenuwein, T. 1997. Mammalian homologues of the Polycomb-group gene Enhancer of zeste mediate gene silencing in Drosophila heterochromatin and at S. cerevisiae telomeres. EMBO J. 16: 32193232.

Lee, S.R. and Collins, K. 2006. Two classes of endogenous small RNAs in Tetrahymena thermophila. Genes \& Dev. 20: 2833.

Lindroth, A.M., Shultis, D., Jasencakova, Z., Fuchs, J., Johnson, L., Schubert, D., Patnaik, D., Pradhan, S., Goodrich, J., Schubert, I., et al. 2004. Dual histone H3 methylation marks at lysines 9 and 27 required for interaction with CHROMOMETHYLASE3. EMBO J. 23: 4286-4296.

Liu, Y., Mochizuki, K., and Gorovsky, M.A. 2004. Histone H3 lysine 9 methylation is required for DNA elimination in developing macronuclei in Tetrahymena. Proc. Natl. Acad. Sci. 101: 1679-1684.

Madireddi, M.T., Davis, M.C., and Allis, C.D. 1994. Identification of a novel polypeptide involved in the formation of DNA-containing vesicles during macronuclear development in Tetrahymena. Dev. Biol. 165: 418-431.

Madireddi, M.T., Coyne, R.S., Smothers, J.F., Mickey, K.M., Yao, M.C., and Allis, C.D. 1996. Pdd1p, a novel chromodomain-containing protein, links heterochromatin assembly and DNA elimination in Tetrahymena. Cell 87: 75-84.

Malone, C.D., Anderson, A.M., Motl, J.A., Rexer, C.H., and Chalker, D.L. 2005. Germ line transcripts are processed by a Dicer-like protein that is essential for developmentally programmed genome rearrangements of Tetrahymena thermophila. Mol. Cell. Biol. 25: 9151-9164.

Martienssen, R.A. and Moazed, D. 2006. RNAi and heterochromatin assembly. In Epigenetics (eds. C.D. Allis et al.), pp. 151-166. Cold Spring Harbor Laboratory Press, Cold Spring Harbor, NY.

Martin, S.E., Shabanowitz, J., Hunt, D.F., and Marto, J.A. 2000. Subfemtomole MS and MS/MS peptide sequence analysis using nano-HPLC micro-ESI fourier transform ion cyclotron resonance mass spectrometry. Anal. Chem. 72: 4266-4274.

Matzke, M.A. and Birchler, J.A. 2005. RNAi-mediated pathways in the nucleus. Nat. Rev. Genet. 6: 24-35.

Meyer, E. and Chalker, D.L. 2007. Epigenetics of ciliates. In Epigenetics (eds. C.D. Allis et al.), pp. 127-150. Cold Spring Harbor Laboratory Press, Cold Spring Harbor, New York.

Min, J., Zhang, Y., and Xu, R.M. 2003. Structural basis for specific binding of Polycomb chromodomain to histone H3 methylated at Lys 27. Genes \& Dev. 17: 1823-1828.

Mochizuki, K. and Gorovsky, M.A. 2004a. Conjugation-specific small RNAs in Tetrahymena have predicted properties of scan (scn) RNAs involved in genome rearrangement. Genes \& Dev. 18: 2068-2073.

Mochizuki, K. and Gorovsky, M.A. 2004b. Small RNAs in genome rearrangement in Tetrahymena. Curr. Opin. Genet. Dev. 14: 181-187.

Mochizuki, K. and Gorovsky, M.A. 2005. A Dicer-like protein in Tetrahymena has distinct functions in genome rearrangement, chromosome segregation, and meiotic prophase. Genes \& Dev. 19: 77-89.

Mochizuki, K., Fine, N.A., Fujisawa, T., and Gorovsky, M.A. 2002. Analysis of a piwi-related gene implicates small RNAs in genome rearrangement in tetrahymena. Cell 110: 689699.

Muller, J., Hart, C.M., Francis, N.J., Vargas, M.L., Sengupta, A., Wild, B., Miller, E.L., O'Connor, M.B., Kingston, R.E., and Simon, J.A. 2002. Histone methyltransferase activity of a Drosophila Polycomb group repressor complex. Cell 111: 
197-208.

Nakayama, J., Rice, J.C., Strahl, B.D., Allis, C.D., and Grewal, S.I. 2001. Role of histone H3 lysine 9 methylation in epigenetic control of heterochromatin assembly. Science 292: 110-113.

Nikiforov, M.A., Smothers, J.F., Gorovsky, M.A., and Allis, C.D. 1999. Excision of micronuclear-specific DNA requires parental expression of pdd2p and occurs independently from DNA replication in Tetrahymena thermophila. Genes \& Dev. 13: 2852-2862.

Nikiforov, M.A., Gorovsky, M.A., and Allis, C.D. 2000. A novel chromodomain protein, pdd3p, associates with internal eliminated sequences during macronuclear development in Tetrahymena thermophila. Mol. Cell. Biol. 20: 4128-4134.

Noma, K., Sugiyama, T., Cam, H., Verdel, A., Zofall, M., Jia, S., Moazed, D., and Grewal, S.I. 2004. RITS acts in cis to promote RNA interference-mediated transcriptional and posttranscriptional silencing. Nat. Genet. 36: 1174-1180.

Okamoto, I., Otte, A.P., Allis, C.D., Reinberg, D., and Heard, E. 2004. Epigenetic dynamics of imprinted X inactivation during early mouse development. Science 303: 644-649.

Pal-Bhadra, M., Bhadra, U., and Birchler, J.A. 1999. Cosuppression of nonhomologous transgenes in Drosophila involves mutually related endogenous sequences. Cell 99: 35-46.

Pal-Bhadra, M., Bhadra, U., and Birchler, J.A. 2002. RNAi related mechanisms affect both transcriptional and posttranscriptional transgene silencing in Drosophila. Mol. Cell 9: 315327.

Partridge, J.F., Scott, K.S., Bannister, A.J., Kouzarides, T., and Allshire, R.C. 2002. cis-acting DNA from fission yeast centromeres mediates histone $\mathrm{H} 3$ methylation and recruitment of silencing factors and cohesin to an ectopic site. Curr. Biol. 12: $1652-1660$.

Peters, A.H., Kubicek, S., Mechtler, K., O'Sullivan, R.J., Derijck, A.A., Perez-Burgos, L., Kohlmaier, A., Opravil, S., Tachibana, M., Shinkai, Y., et al. 2003. Partitioning and plasticity of repressive histone methylation states in mammalian chromatin. Mol. Cell 12: 1577-1589.

Plath, K., Fang, J., Mlynarczyk-Evans, S.K., Cao, R., Worringer, K.A., Wang, H., de la Cruz, C.C., Otte, A.P., Panning, B., and Zhang, Y. 2003. Role of histone H3 lysine 27 methylation in $\mathrm{X}$ inactivation. Science 300: 131-135.

Ringrose, L. and Paro, R. 2004. Epigenetic regulation of cellular memory by the Polycomb and Trithorax group proteins. Annu. Rev. Genet. 38: 413-443.

Shang, Y., Song, X., Bowen, J., Corstanje, R., Gao, Y., Gaertig, J., and Gorovsky, M.A. 2002. A robust inducible-repressible promoter greatly facilitates gene knockouts, conditional expression, and overexpression of homologous and heterologous genes in Tetrahymena thermophila. Proc. Natl. Acad. Sci. 99: 3734-3739.

Silva, J., Mak, W., Zvetkova, I., Appanah, R., Nesterova, T.B., Webster, Z., Peters, A.H., Jenuwein, T., Otte, A.P., and Brockdorff, N. 2003. Establishment of histone h3 methylation on the inactive $\mathrm{X}$ chromosome requires transient recruitment of Eed-Enx1 polycomb group complexes. Dev. Cell 4: 481-495.

Smothers, J.F., Mizzen, C.A., Tubbert, M.M., Cook, R.G., and Allis, C.D. 1997. Pdd1p associates with germline-restricted chromatin and a second novel anlagen-enriched protein in developmentally programmed DNA elimination structures. Development 124: 4537-4545.

Strahl, B.D., Ohba, R., Cook, R.G., and Allis, C.D. 1999. Methylation of histone $\mathrm{H} 3$ at lysine 4 is highly conserved and correlates with transcriptionally active nuclei in Tetrahymena. Proc. Nat1. Acad. Sci. 96: 14967-14972.
Sung, S. and Amasino, R.M. 2004. Vernalization in Arabidopsis thaliana is mediated by the PHD finger protein VIN3. Nature 427: 159-164.

Syka, J.E., Marto, J.A., Bai, D.L., Horning, S., Senko, M.W., Schwartz, J.C., Ueberheide, B., Garcia, B., Busby, S., Muratore, T., et al. 2004. Novel linear quadrupole ion trap/FT mass spectrometer: Performance characterization and use in the comparative analysis of histone $\mathrm{H} 3$ post-translational modifications. J. Proteome Res. 3: 621-626.

Tagami, H., Ray-Gallet, D., Almouzni, G., and Nakatani, Y. 2004. Histone H3.1 and H3.3 complexes mediate nucleosome assembly pathways dependent or independent of DNA synthesis. Cell 116: 51-61.

Taverna, S.D., Coyne, R.S., and Allis, C.D. 2002. Methylation of histone h3 at lysine 9 targets programmed DNA elimination in tetrahymena. Cell 110: 701-711.

Taverna, S.D., Ueberheide, B.M., Liu, Y., Tackett, A.J., Diaz, R.L., Shabanowitz, J., Chait, B.T., Hunt, D.F., and Allis, C.D. 2007. Long-distance combinatorial linkage between methylation and acetylation on histone $\mathrm{H} 3 \mathrm{~N}$ termini. Proc. Nat1. Acad. Sci. 104: 2086-2091.

Vavra, K.J., Allis, C.D., and Gorovsky, M.A. 1982. Regulation of histone acetylation in Tetrahymena macro- and micronuclei. J. Biol. Chem. 257: 2591-2598.

Verdel, A., Jia, S., Gerber, S., Sugiyama, T., Gygi, S., Grewal, S.I., and Moazed, D. 2004. RNAi-mediated targeting of heterochromatin by the RITS complex. Science 303: 672-676.

Volpe, T.A., Kidner, C., Hall, I.M., Teng, G., Grewal, S.I., and Martienssen, R.A. 2002. Regulation of heterochromatic silencing and histone H3 lysine-9 methylation by RNAi. Science 297: 1833-1837. 


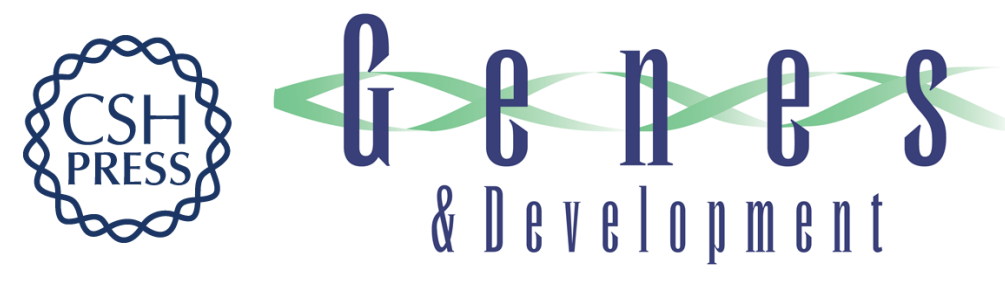

\section{RNAi-dependent H3K27 methylation is required for heterochromatin formation and DNA elimination in Tetrahymena}

Yifan Liu, Sean D. Taverna, Tara L. Muratore, et al.

Genes Dev. 2007, 21:

Access the most recent version at doi:10.1101/gad.1544207

Supplemental
Material http://genesdev.cshlp.org/content/suppl/2007/06/12/21.12.1530.DC1

References This article cites 77 articles, 40 of which can be accessed free at:

http://genesdev.cshlp.org/content/21/12/1530.full.html\#ref-list-1

License

Email Alerting Receive free email alerts when new articles cite this article - sign up in the box at the top

Service

right corner of the article or click here.

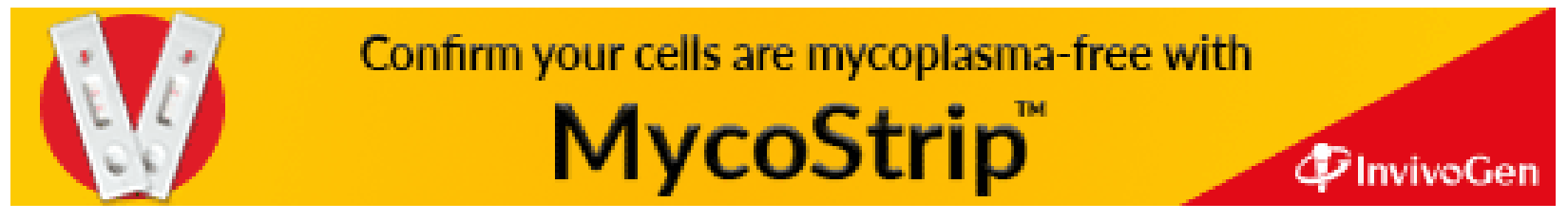

